\title{
A FINITE VOLUME SCHEME FOR THE SOLUTION OF A MIXED DISCRETE-CONTINUOUS FRAGMENTATION MODEL
}

\author{
GRAHAM BAIRD* AND ENDRE SÜLI
}

\begin{abstract}
This paper concerns the construction and analysis of a numerical scheme for a mixed discrete-continuous fragmentation equation. A finite volume scheme is developed, based on a conservative formulation of a truncated version of the equations. The approximate solutions provided by this scheme are first shown to display conservation of mass and preservation of nonnegativity. Then, by utilising a Dunford-Pettis style argument, the sequence of approximate solutions generated is shown, under given restrictions on the model and the mesh, to converge (weakly) in an appropriate $L_{1}$ space to a weak solution to the problem. By applying the methods and theory of operator semigroups, we are able to show that these weak solutions are unique and necessarily classical (differentiable) solutions, a degree of regularity not generally established when finite volume schemes are applied to such problems. Furthermore, this approach enabled us to derive a bound for the error induced by the truncation of the mass domain, and also establish the convergence of the truncated solutions as the truncation point is increased without bound. Finally, numerical simulations are performed to investigate the performance of the scheme and assess its rate of convergence as the mesh is refined, whilst also verifying the bound on the truncation error.
\end{abstract}

Mathematics Subject Classification. 35R09, 47D06, 65M08.

Received February 2, 2019. Accepted December 17, 2020.

\section{INTRODUCTION}

Fragmentation and coagulation processes occur in many physical systems, with the associated mathematical models receiving much attention in the literature. Example application areas include colloid science $[20,55]$, population dynamics $[21,22]$ and astrophysics [23,32]. However, analytical solutions to these models are only available for a limited number of specific cases, and we often have to resort to approximate solutions generated by an appropriate numerical scheme. A range of numerical techniques have been applied to these problems, and these broadly fall into two categories: those involving a stochastic (Monte Carlo) element, for example $[3,24,31]$ and those based around various deterministic approximation schemes [16,18,26,27,30,33-37,46,48-51]. The introductory chapter of [34] and the references therein provide a detailed overview of a number of these approximation methods.

In the earlier work [5], we presented a mixed discrete-continuous model of fragmentation in an attempt to resolve the issue of "shattering" mass-loss observed in some purely continuous models [28,42]. By modelling the

Keywords and phrases. Fragmentation equations, finite volume scheme, mixed discrete-continuous fragmentation model.

Mathematical Institute, University of Oxford, Oxford OX2 6GG, UK.

*Corresponding author: graham.baird@maths.ox.ac.uk 
mass distribution amongst the smallest particles using a discrete model, whilst modelling the distribution of larger particle masses with a continuous model, the aim was to introduce a higher degree of physical fidelity thus resolving the shattering mass-loss problem, whilst also retaining the mathematical efficiency of the continuous model.

Given the similarities between this model and those existing in the literature, in addition to the added complexity of the mixed framework, we would expect in most cases to have to rely on numerical methods to obtain a solution. In this paper we present a numerical scheme for the solution of the mixed fragmentation model proposed in [5]. The basis of the scheme is a finite volume discretisation of the continuous regime equation. The use of such a method would appear a reasonable choice in this case, given its conservative nature and the motivation behind the model development. Indeed, finite volume schemes have been commonly applied to the solution of coagulation and fragmentation equations, with the first such use being [27], where the case of pure coagulation was considered. For problems involving fragmentation, the article [18] sees such a scheme employed in approximating the binary coagulation and fragmentation equation, whilst [33,34] examine their use for the multiple fragmentation equation, with [37] extending this to include coagulation. Further works have seen these methods applied to a number of coagulation-fragmentation model variants, for example with the inclusion of spatial diffusion [26] and additional nucleation and growth processes [36,49]. Whilst a number of articles $[30,48,50]$ cover the approximation of multi-dimensional coagulation or fragmentation, whereby particles may be classified by additional variables beyond their mass or volume.

\subsection{Mixed discrete-continuous model}

In the mixed model of [5], a cut-off value $N \in \mathbb{N}$ is introduced; above this cut-off, particle mass is considered as a continuous variable, whilst below it, the particles are forced to take discrete integer masses. If we denote by $u_{C}(x, t)$ the particle mass density within the continuous mass regime $(x>N)$, then the evolution of $u_{C}(x, t)$ is governed by the continuous multiple fragmentation equation:

$$
\begin{aligned}
\frac{\partial u_{C}(x, t)}{\partial t} & =-a(x) u_{C}(x, t)+\int_{x}^{\infty} a(y) b(x \mid y) u_{C}(y, t) \mathrm{d} y, \quad x>N, \quad t>0 \\
u_{C}(x, 0) & =c_{0}(x) .
\end{aligned}
$$

This equation is similar in form to the multiple fragmentation equation introduced in [42]. The function $a(x)$ provides the fragmentation rate for a particle of mass $x$, whilst $b(x \mid y)$ represents the distribution of particles of mass $x>N$ resulting from the break-up of a particle of mass $y>x$. The functions $a$ and $b$ are assumed to be nonnegative measurable functions, defined on $(N, \infty)$ and $(N, \infty) \times(N, \infty)$, respectively. We also require $b(x \mid y)=0$ for $x>y$, since no particle resulting from a fragmentation event can have a mass exceeding the original particle. The initial mass distribution within the continuous regime is given by the nonnegative function $c_{0}(x)$.

Letting $u_{D i}(t)$ denote the concentration of discrete mass $i$-mer particles $(i \leq N)$ and $u_{D}(t)$ the $N$-component vector taking these values as entries, the change in the values $u_{D i}(t), i=1, \ldots, N$, is governed by the equation:

$$
\begin{aligned}
\frac{\mathrm{d} u_{D i}(t)}{\mathrm{d} t} & =-a_{i} u_{D i}(t)+\sum_{j=i+1}^{N} a_{j} b_{i, j} u_{D j}(t)+\int_{N}^{\infty} a(y) b_{i}(y) u_{C}(y, t) \mathrm{d} y, t>0, \\
u_{D}(0) & =d_{0} .
\end{aligned}
$$

In the case of $i=N$, the second term becomes an empty sum and is taken to be 0 . The values $a_{i}$ give the rates at which $i$-mer particles fragment, with $a_{1}=0$. The quantities $b_{i, j}$ give the expected number of $i$-mers produced from the fragmentation of a $j$-mer and the functions $b_{i}(y)$ give the expected number of $i$-mers produced from the fragmentation of a particle of mass $y>N$. The underlying physics demands that each $a_{i}, b_{i, j}$ and $b_{i}(y)$ be nonnegative. Finally, $d_{0}$ is the $N$-component vector of nonnegative values, specifying the initial concentrations within the discrete regime. 
During each fragmentation event, mass is simply redistributed from the larger particle to the smaller resulting particles, but the total mass involved should be conserved. This gives rise to the following two conditions to supplement equations (1.1) and (1.2):

$$
\begin{aligned}
\int_{N}^{y} x b(x \mid y) \mathrm{d} x+\sum_{j=1}^{N} j b_{j}(y) & =y \quad \text { for } y>N, \\
\sum_{j=1}^{i-1} j b_{j, i} & =i \quad \text { for } i=2, \ldots, N .
\end{aligned}
$$

The condition (1.3) is an expression of mass conservation upon the fragmentation of a particle from the continuous mass regime. The equation (1.4) comes from the conservation of mass when a particle from the discrete mass regime breaks up.

For further details on the mixed discrete-continuous model and its properties, the reader is directed to consult [5] or [4].

\subsection{Truncation and reformulation}

When considering the numerical solution of equations (1.1) and (1.2), we encounter an issue in that the range of the continuous mass variable $x$ is an unbounded interval, which presents a computational problem. We therefore introduce a truncation parameter $R>N$, and restrict the continuous mass variable to the range $N<x<R$. Therefore, in place of equations (1.1), we consider the truncated version

$$
\begin{aligned}
\frac{\partial u_{C}^{R}(x, t)}{\partial t} & =-a(x) u_{C}^{R}(x, t)+\int_{x}^{R} a(y) b(x \mid y) u_{C}^{R}(y, t) \mathrm{d} y, \quad N<x<R, t>0 \\
u_{C}^{R}(x, 0) & =\chi_{(N, R)}(x) c_{0}(x), \quad N<x<R
\end{aligned}
$$

where $\chi_{(N, R)}$ denotes the characteristic function of the interval $(N, R)$.

Taking our lead from the aforementioned articles, we now rewrite equation (1.5) in a conservative form, although in our case we must include an additional sink term to account for the mass leaked down to the discrete regime. Therefore we end up with the following equation for the mass quantity $x u_{C}^{R}(x, t)$ :

$$
\frac{\partial\left(x u_{C}^{R}\right)}{\partial t}=\frac{\partial \mathcal{F}^{R}\left(x u_{C}^{R}\right)}{\partial x}-S\left(x u_{C}^{R}\right), u_{C}^{R}(x, 0)=c_{0}(x), \text { for } N<x<R, t>0,
$$

where $\mathcal{F}^{R}$ and $S$ are a truncated flux term and sink term, respectively, given by

$$
\mathcal{F}^{R}(f)(x)=\int_{x}^{R} \int_{N}^{x} \frac{y}{z} a(z) b(y \mid z) f(z) \mathrm{d} y \mathrm{~d} z, \quad S(f)(x)=\frac{a(x)}{x} \sum_{i=1}^{N} i b_{i}(x) f(x), \text { for } N<x<R .
$$

The equation (1.5) may be recovered from (1.6) by a formal application of Leibniz's rule for differentiating under the integral. However, the equivalence of the two forms can be seen to be justified rigorously in Appendix $\mathrm{C}$ of [4]. Before continuing, we establish a result concerning the behaviour of the flux term $\mathcal{F}^{R}$ at the limits of our domain.

Lemma 1.1. If the kernels $a$ and $b$ are assumed to belong to $L_{\infty, \text { loc }}$ on the domains $[N, \infty)$ and $[N, \infty) \times[N, \infty)$ respectively, which will be the case in the upcoming analysis, then for $f \in L_{1}(N, R)$ the flux term $\mathcal{F}^{R}(f)$ satisfies

$$
\lim _{x \rightarrow N, R}\left|\mathcal{F}^{R}(f)(x)\right|=0 .
$$


Proof. It is a straightforward matter to bound $\mathcal{F}^{R}(f)$ as follows:

$$
\left|\mathcal{F}^{R}(f)(x)\right| \leq \int_{N}^{R} \chi_{(x, R)}(z) \frac{a(z)|f(z)|}{z}\left(\int_{N}^{x} y b(y \mid z) \mathrm{d} y\right) \mathrm{d} z
$$

which holds for $x \in(N, R)$. Recalling the mass conservation condition (1.3), we deduce that

$$
\chi_{(x, R)}(z)\left(\int_{N}^{x} y b(y \mid z) \mathrm{d} y\right) \leq \int_{N}^{z} y b(y \mid z) \mathrm{d} y \leq z,
$$

for all $z \in(N, R)$. Hence the integrand appearing in (1.7) is bounded above by $a(z)|f(z)|$, which, thanks to $a \in L_{\infty, \text { loc }}[N, \infty)$ and $f \in L_{1}(N, R)$, is integrable.

Considering the limit as $x \rightarrow N$ first, if we denote by $\beta(R)$ the essential supremum of $b$ over $[N, R] \times[N, R]$, then we have

$$
\chi_{(x, R)}(z)\left(\int_{N}^{x} y b(y \mid z) \mathrm{d} y\right) \leq x \beta(R)(x-N) .
$$

As such, the integrand in (1.7) converges pointwise to 0 over $z \in(N, R)$ as we let $x \searrow N$. An application of the Lebesgue dominated convergence theorem then gives the required convergence of $\left|\mathcal{F}^{R}(f)(x)\right|$ as $x \searrow N$. Turning now to the limit as $x \nearrow R$, another application of condition (1.3) provides us with

$$
\chi_{(x, R)}(z)\left(\int_{N}^{x} y b(y \mid z) \mathrm{d} y\right) \leq \chi_{(x, R)}(z) \int_{N}^{z} y b(y \mid z) \mathrm{d} y \leq \chi_{(x, R)}(z) z
$$

for $z \in(N, R)$. Therefore, the integrand from (1.7) must again converge pointwise to 0 over $(N, R)$, this time as we let $x \nearrow R$. Another application of the Lebesgue dominated convergence theorem gives the convergence of $\left|\mathcal{F}^{R}(f)(x)\right|$ to 0 , as $x \nearrow R$.

This result will be utilised later in a number of arguments, most significantly in approximating $\mathcal{F}^{R}$ within our numerical scheme and in establishing a weak formulation of equation (1.6).

Remark 1.2. Additionally we might note that $\mathcal{F}^{R}(f) \in W_{1}^{1}((N, R))$. In the upcoming Definition 5.5 we introduce a weak formulation of (1.6), this observation regarding $\mathcal{F}^{R}(f)$ would permit an alternative formulation without the requirement for an $x$-derivative.

The truncation of the continuous mass interval also has an impact on our discrete regime equation; therefore, instead of equation (1.2), we consider

$$
\begin{aligned}
\frac{\mathrm{d} u_{D i}^{R}(t)}{\mathrm{d} t} & =-a_{i} u_{D i}^{R}(t)+\sum_{j=i+1}^{N} a_{j} b_{i, j} u_{D j}^{R}(t)+\int_{N}^{R} a(y) b_{i}(y) u_{C}^{R}(y, t) \mathrm{d} y, \\
u_{D i}^{R}(0) & =d_{0 i}, \text { for } i=1,2, \ldots, N, \quad t>0 .
\end{aligned}
$$

In the case of $i=N$, the empty sum above is taken to be zero; this convention will be adopted in all similar cases which follow.

This truncation procedure is a standard approach when dealing with fragmentation and coagulation problems, having been applied for example in [44], where the theory and methods of operator semigroups were employed, and [53] where an alternative weak compactness style argument was adopted. The common approach of these works involves establishing the existence of solutions to a sequence of such truncated problems. A limit is then obtained as the truncation point is increased without bound, with this limit then being shown to satisfy the untruncated problem in some sense. 


\section{Preliminaries}

Having set out our problem in the previous section, we now present a brief outline of the key results which appear in the upcoming material and which may be considered nonstandard or which are particular to our case.

Theorem 2.1. In the analysis pursued in subsequent results, we shall be working extensively in spaces of the type $L_{1}$. In particular we shall be working in the spaces $L_{1}=L_{1}((N, R) \times[0, T), \mathrm{d} x \mathrm{~d} t)$ and $L_{1}^{1}=L_{1}((N, R) \times$ $[0, T), x \mathrm{~d} x \mathrm{~d} t)$, where $N$ is a positive integer and $R>N$ is a finite real value. With the associated norms, these form equivalent spaces.

Proof. The proof of this relies on the boundedness of the mass domain $N<x<R$ in order to bound the weighting applied to the measure $\mathrm{d} x$. The details of this procedure are straightforward, and therefore a full proof is omitted. However, full details of the derivation can be found in Theorem 2.1.10 of [4].

This result shall prove useful in the forthcoming analysis, allowing us to switch spaces when mathematically convenient whilst retaining convergence.

Given a sequence $\left\{f_{n}\right\}_{n=1}^{\infty}$ in a normed vector space $(X,\|\cdot\|)$, we assume the reader is familiar with the concept of weak convergence and in particular its definition in spaces of the type $L_{1}(\Omega, \mu)$. In our analysis when handling weakly convergent sequences we will usually find them appearing alongside other factors and we would like the product to converge weakly also. The following theorem gives us sufficient conditions for the product of two sequences to converge weakly and will be used extensively in the convergence proofs for our numerical schemes.

Theorem 2.2. Let $(\Omega, \mathcal{A}, \mu)$ be a measure space with $\mu$ finite. Suppose $f_{h} \rightarrow f$ in $L_{1}(\Omega, \mu), g_{h} \rightarrow g$ point-wise $\mu$ a.e. in $\Omega$, and $\sup _{h}\left\|g_{h}\right\|_{L_{\infty}}<\infty$, then $f_{h} g_{h} \rightarrow f g$ in $L_{1}(\Omega, \mu)$.

Proof. The reader is referred to Proposition 2.61 of [29].

The main part of our convergence argument utilises the Dunford-Pettis theorem, which provides us with sufficient conditions to establish the weak convergence of our sequence of approximations. One such condition is that of equiintegrability. There are a number of equivalent characterisations of equiintegrability, which the reader may find in Theorem 2.29 of [29]. For our purposes the most important characterisation of equiintegrability is given by de la Vallée Poussin's theorem, a refined version of which is given below.

Theorem 2.3. (de la Vallée Poussin's Theorem) Let $\mathcal{F}$ be a bounded subset of $L_{1}(\Omega, \mu)$; then $\mathcal{F}$ is equiintegrable if and only if there exists a nonnegative, convex function $\Phi \in C^{\infty}([0, \infty))$, with $\Phi(0)=0$ and $\Phi^{\prime}(0)=1$, such that $\Phi^{\prime}$ is concave and

$$
\frac{\Phi(x)}{x} \rightarrow \infty \text { as } x \rightarrow \infty \text { and } \sup _{f \in \mathcal{F}} \int_{\Omega} \Phi(|f|) \mathrm{d} \mu<\infty .
$$

Proof. The necessity of this condition can be derived easily from Theorem 8 of [39], which under the assumption that $\mathcal{F}$ is equiintegrable provides us with a $\Psi$ satisfying all the stated conditions with the exception that the derivative of $\Psi$ takes the value 0 at 0 . Given such a $\Psi$, we set $\Phi(x)=\Psi(x)+x$. Then $\Phi$ retains the required properties of $\Psi$ but additionally $\Phi^{\prime}(0)=1$. Also, by utilising the following standard inequality for $C^{1}$ convex functions

$$
\Phi(x) \geq \Phi(y)+\Phi^{\prime}(y)(x-y),
$$

with $x \geq 0$ and $y=0$ we can see that $\Phi(x)$ must be nonnegative on $[0, \infty)$. The sufficiency of our conditions comes straight from the standard version of the de la Vallée Poussin theorem ([29], Thm. 2.29(iii)).

In our analysis we shall require some properties of such a function, which we set out in the following lemma.

Lemma 2.4. Let $\Phi$ be as in Theorem 2.3; then for nonnegative $x$ and $y$ we have the following: 
(1) $x \Phi^{\prime}(y) \leq \Phi(x)+\Phi(y)$,

(2) $\Phi^{\prime}(y) \geq 1$.

Proof. The first of these inequalities is nonstandard and the proof can be found in Proposition 14 of [39]. The second property is readily derived from the properties required by Theorem 2.3. As a convex $C^{2}$ function we necessarily have that $\Phi^{\prime \prime} \geq 0$, hence $\Phi^{\prime}$ is a nondecreasing function and $\Phi^{\prime}(x) \geq \Phi^{\prime}(0)=1$, for all $x \geq 0$.

We now come to the Dunford-Pettis theorem, one of the most significant technical tools applied in this work. The theorem provides necessary and sufficient conditions for a subset of an $L_{1}$ space to be weakly sequentially compact. That is, any sequence in the subset must have a subsequence which is weakly convergent.

Theorem 2.5. (Dunford-Pettis Theorem) Let $(\Omega, \mathcal{A}, \mu)$ be a measure space and let $\mathcal{F} \subset L_{1}(\Omega, \mu)$. Then $\mathcal{F}$ is weakly sequentially compact if and only if the following conditions are satisfied:

(1) $\mathcal{F}$ is bounded in $L_{1}(\Omega, \mu)$;

(2) $\mathcal{F}$ is equiintegrable;

(3) For every $\varepsilon>0$ there exists $A_{\varepsilon} \subset \Omega$ with $A_{\varepsilon} \in \mathcal{A}$ such that $\mu\left(A_{\varepsilon}\right)<\infty$ and

$$
\sup _{f \in \mathcal{F}} \int_{\Omega \backslash A_{\varepsilon}}|f| \mathrm{d} \mu \leq \varepsilon
$$

We note that in the case that $\mu(\Omega)<\infty$ condition (iii) is automatically satisfied by taking $A_{\varepsilon}=\Omega$ for all values of $\varepsilon$.

Proof. See Theorem 2.54 of [29].

In the later analysis of this paper we shall be relying heavily on the methods and theory of operator semigroups. In particular the concept of substochastic semigroups, the Kato-Voigt perturbation theorem and the notion of semigroup honesty. For the sake of brevity we refrain from outlining such material here, however the reader may find details of the requisite results in the preliminary sections of [5] or [4] or the text [8].

\section{DeVElopment of the NUMERICAL SCHEME}

We now introduce our numerical approximation scheme for the truncated system, (1.6) and (1.8). First we must discretise the continuous mass variable $x$, and so we introduce the mesh $\left\{x_{i-1 / 2}\right\}_{i=0}^{I_{h}}$ on the interval $(N, R)$, with

$$
x_{-1 / 2}=N, x_{I_{h}-1 / 2}=R, x_{i}=\left(x_{i-1 / 2}+x_{i+1 / 2}\right) / 2, h / k<\Delta x_{i}=x_{i+1 / 2}-x_{i-1 / 2}<h,
$$

where $h \in(0,1)$ and $k>1$ is some constant. Additionally we denote the interval $\left[x_{i-1 / 2}, x_{i+1 / 2}\right)$ by $\Lambda_{i}$, however the (left-hand-most) interval $\Lambda_{0}$ is taken to be $\left(x_{-1 / 2}, x_{1 / 2}\right)$.

For the time variable $t$, if $T$ is the final time up to which we wish to compute an approximate solution, then we define the time step $\Delta t=T / M$ where $M$ is some large integer. The time points are then given by $t_{n}=n \Delta t$ for $n=0,1, \ldots, M$ with corresponding time intervals $\tau_{n}=\left[t_{n}, t_{n+1}\right)$ for $n=0,1, \ldots, M-1$.

We restrict the choice of the mesh by assuming the existence of positive constants $k_{1}$ and $k_{2}$ so that the mesh sizes $h$ and $\Delta t$ satisfy

$$
k_{1} h \leq \Delta t \leq k_{2} h .
$$

The numerical scheme requires representative values for the functions $a(x), b(x \mid y)$ and $b_{i}(y)$ over the appropriate intervals. This is done by taking their average value over each interval. Therefore we define

$$
A_{i}=\frac{1}{\Delta x_{i}} \int_{\Lambda_{i}} a(x) \mathrm{d} x \text { for } i=0,1, \ldots, I_{h}-1,
$$


as our approximation of $a(x)$ over the interval $\Lambda_{i}$. We approximate $b(x \mid y)$ over $\Lambda_{i} \times \Lambda_{j}$ by

$$
B_{i, j}=\frac{1}{\Delta x_{i} \Delta x_{j}} \int_{\Lambda_{j}} \int_{\Lambda_{i}} b(x \mid y) \mathrm{d} x \mathrm{~d} y \text { for } i=0,1, \ldots, I_{h}-1 \text { and } j=0,1, \ldots, I_{h}-1,
$$

and the functions $b_{i}(y)$ are approximated over $\Lambda_{j}$ by the values

$$
\tilde{B}_{i, j}=\frac{1}{\Delta x_{j}} \int_{\Lambda_{j}} b_{i}(y) \mathrm{d} y \text { for } i=1,2, \ldots, N \text { and } j=0,1, \ldots, I_{h}-1 .
$$

We note by our initial assumption regarding the nonnegativity of $a, b$ and $b_{i}$, that each of the values introduced above must be nonnegative. If $\chi_{I}$ denotes the characteristic function of a set $I$, then we can construct piecewise constant approximations to the functions $a, b$ and $b_{i}$ as follows:

$$
a^{h}(x)=\sum_{i=0}^{I_{h}-1} \chi_{\Lambda_{i}}(x) A_{i}, \quad b^{h}(x \mid y)=\sum_{j=0}^{I_{h}-1} \sum_{i=0}^{I_{h}-1} \chi_{\Lambda_{i}}(x) \chi_{\Lambda_{j}}(y) B_{i, j}, \quad b_{i}^{h}(y)=\sum_{j=0}^{I_{h}-1} \chi_{\Lambda_{j}}(y) \tilde{B}_{i, j} .
$$

Remark 3.1. This is a standard means of approximation and assuming the choice of kernels is suitably restricted, the approximations will converge pointwise to the desired functions almost everywhere on the appropriate domains. In our case, the kernels $a$ and $b$ will be assumed to be $L_{\infty, \text { loc }}$ on $[N, \infty)$ and $[N, \infty) \times[N, \infty)$, respectively. In addition, the restriction (1.3) determines each $b_{i}$ as an element of $L_{\infty, \text { loc }}[N, \infty)$. Having $a, b$ and $b_{i}$ as $L_{\infty, \text { loc }}$ functions is sufficient to ensure that the approximations $a^{h}, b^{h}$ and $b_{i}^{h}$ converge pointwise to $a$, $b$ and $b_{i}$ almost everywhere in their respective domains. This is a standard result, however full details can be found in Lemma 4.2.1 of [4].

We are now ready to construct the approximation scheme. Let $u_{C}^{n, i}$ denote our approximation to $u_{C}^{R}(x, t)$ over the mass interval $\Lambda_{i}$ for the time interval $\tau_{n}$. The equation (1.6) is then approximated by

$$
x_{i} \frac{u_{C}^{n+1, i}-u_{C}^{n, i}}{\Delta t}=\frac{F_{i+1 / 2}^{n}-F_{i-1 / 2}^{n}}{\Delta x_{i}}-S_{i}^{n},
$$

where $F_{i-1 / 2}^{n}$ is an approximation of the flux $\mathcal{F}^{R}\left(x u_{C}^{R}\right)$ at the point $x=x_{i-1 / 2}$ over the time interval $\tau_{n}$, and is given by

$$
\begin{aligned}
\left(\mathcal{F}^{R}\left(x u_{C}^{R}\right)\right)\left(x_{i-1 / 2}\right) & =\int_{x_{i-1 / 2}}^{R} \int_{N}^{x_{i-1 / 2}} y a(z) b(y \mid z) u_{C}^{R}(z, t) \mathrm{d} y \mathrm{~d} z \\
& =\sum_{j=i}^{I_{h}-1} \int_{\Lambda_{j}}\left(\sum_{k=0}^{i-1} \int_{\Lambda_{k}} y a(z) b(y \mid z) u_{C}^{R}(z, t) \mathrm{d} y\right) \mathrm{d} z \\
& \approx \sum_{j=i}^{I_{h}-1} \sum_{k=0}^{i-1} x_{k} A_{j} B_{k, j} u_{C}^{n, j} \Delta x_{k} \Delta x_{j}=: F_{i-1 / 2}^{n},
\end{aligned}
$$

for $i=1, \ldots, I_{h}-1$, with $F_{-1 / 2}^{n}=F_{I_{h}-1 / 2}^{n}=0$, which can be justified by Lemma 1.1. The values $S_{i}^{n}$ approximate the sink term $S\left(x u_{C}^{R}\right)$ over $\Lambda_{i}$ for the time interval $\tau_{n}$, and are computed by

$$
S_{i}^{n}=A_{i} \sum_{j=1}^{N} j \tilde{B}_{j, i} u_{C}^{n, i} \text { for } i=0,1, \ldots, I_{h}-1 .
$$

This gives rise to the following numerical method for the computation of the approximations $u_{C}^{n, i}$ :

$$
u_{C}^{n+1, i}=u_{C}^{n, i}+\frac{\Delta t}{x_{i} \Delta x_{i}}\left(F_{i+1 / 2}^{n}-F_{i-1 / 2}^{n}\right)-\frac{\Delta t}{x_{i}} S_{i}^{n} \text { for }\left\{\begin{array}{l}
i=0,1, \ldots, I_{h}-1 \\
n=0,1, \ldots, M-1
\end{array}\right.
$$


The sequence of approximations generated by (3.3) requires us to provide an initial set of values to get started. For our starting values we simply average the initial datum over each of the mass intervals; hence

$$
u_{C}^{0, i}=\frac{1}{\Delta x_{i}} \int_{\Lambda_{i}} c_{0}(x) \mathrm{d} x \text { for } i=0,1, \ldots, I_{h}-1 .
$$

Then our approximation to $u_{C}^{R}(x, t)$ over $(N, R) \times[0, T)$ is constructed as follows:

$$
u_{C}^{h}(x, t)=\sum_{n=0}^{M-1} \sum_{i=0}^{I_{h}-1} \chi_{\Lambda_{i}}(x) \chi_{\tau_{n}}(t) u_{C}^{n, i}
$$

Now considering the discrete regime, let $u_{D}^{n, i}$ denote our approximation of $u_{D i}^{R}(t)$ over the time interval $\tau_{n}$. Equation (1.8) is then approximated as

$$
\frac{u_{D}^{n+1, i}-u_{D}^{n, i}}{\Delta t}=-a_{i} u_{D}^{n, i}+\sum_{j=i+1}^{N} a_{j} b_{i, j} u_{D}^{n, j}+\sum_{j=0}^{I_{h}-1} A_{j} \tilde{B}_{i, j} u_{C}^{n, j} \Delta x_{j}
$$

giving rise to the relation

$$
u_{D}^{n+1, i}=\left(1-\Delta t a_{i}\right) u_{D}^{n, i}+\Delta t \sum_{j=i+1}^{N} a_{j} b_{i, j} u_{D}^{n, j}+\Delta t \sum_{j=0}^{I_{h}-1} A_{j} \tilde{B}_{i, j} u_{C}^{n, j} \Delta x_{j} \text { for }\left\{\begin{array}{l}
i=1, \ldots, N, \\
n=0,1, \ldots, M-1 .
\end{array}\right.
$$

The initial values for the discrete approximation are simply given by the initial condition vector $d_{0}$, so that $u_{D}^{0, i}=d_{0 i}$ for $i=1, \ldots, N$. Then our approximations $u_{D i}^{h}(t)$ to $u_{D i}^{R}(t)$ for $t \in[0, T)$ are given by

$$
u_{D i}^{h}(t)=\sum_{n=0}^{M-1} \chi_{\tau_{n}}(t) u_{D}^{n, i} \quad \text { for } i=1,2, \ldots, N .
$$

\section{Properties of NUMERICAL SOLUtions: NONNEGATIVITY AND MASS CONSERVATION}

In the article [5] we proved the existence and uniqueness of a solution to our system (1.1) and (1.2). This solution was shown to possess a number of properties that we would expect given the physical nature of the model, namely the solution preserves nonnegativity and conserves total mass. In the following sections we examine whether the approximate solution provided by (3.4) and (3.6), also displays these properties. These properties, apart from being physically relevant, will also be utilised in the forthcoming proofs of the convergence of the approximations (3.4) and (3.6) to a solution to the system (1.5) and (1.8), and subsequently the uniqueness and differentiability of that solution.

\subsection{Nonnegativity of the numerical solution}

Lemma 4.1. For a fixed partition $\left(x_{i-1 / 2}\right)_{i=0}^{I_{h}}$, suppose that $\Delta t$ is sufficiently small that the following condition is satisfied:

$$
0<\Delta t \leq \frac{x_{i}}{A_{i}\left(\sum_{k=0}^{i-1} x_{k} B_{k, i} \Delta x_{k}+\sum_{j=1}^{N} j \tilde{B}_{j, i}\right)},
$$

for all $i \in\left\{0,1, \ldots, I_{h}-1\right\}$ such that the denominator is nonzero, and

$$
0<\Delta t \leq \frac{1}{a_{i}}
$$

for all $i \in\{2, \ldots, N\}$ such that $a_{i} \neq 0$. Then, the approximate solutions defined by (3.4) and (3.6) preserve nonnegativity. 
Proof. Starting with the approximation for the continuous regime, let us consider equation (3.3). By cancelling common terms we get that

$$
F_{i+1 / 2}^{n}-F_{i-1 / 2}^{n}=x_{i} \Delta x_{i} \sum_{j=i+1}^{I_{h}-1} A_{j} B_{i, j} u_{C}^{n, j} \Delta x_{j}-A_{i} u_{C}^{n, i} \Delta x_{i} \sum_{k=0}^{i-1} x_{k} B_{k, i} \Delta x_{k}
$$

for $i=1, \ldots, I_{h}-2$. Therefore we have

$$
\begin{aligned}
\frac{\Delta t}{x_{i} \Delta x_{i}}\left(F_{i+1 / 2}^{n}-F_{i-1 / 2}^{n}\right)-\frac{\Delta t}{x_{i}} S_{i}^{n} & =\Delta t \sum_{j=i+1}^{I_{h}-1} A_{j} B_{i, j} u_{C}^{n, j} \Delta x_{j}-\frac{\Delta t}{x_{i}} A_{i} u_{C}^{n, i} \sum_{k=0}^{i-1} x_{k} B_{k, i} \Delta x_{k}-\frac{\Delta t}{x_{i}} A_{i} u_{C}^{n, i} \sum_{j=1}^{N} j \tilde{B}_{j, i} \\
& =\Delta t \sum_{j=i+1}^{I_{h}-1} A_{j} B_{i, j} u_{C}^{n, j} \Delta x_{j}-\frac{\Delta t}{x_{i}} A_{i} u_{C}^{n, i}\left(\sum_{k=0}^{i-1} x_{k} B_{k, i} \Delta x_{k}+\sum_{j=1}^{N} j \tilde{B}_{j, i}\right) .
\end{aligned}
$$

Substituting this into (3.3) gives us

$$
u_{C}^{n+1, i}=\left(1-\frac{\Delta t}{x_{i}} A_{i}\left(\sum_{k=0}^{i-1} x_{k} B_{k, i} \Delta x_{k}+\sum_{j=1}^{N} j \tilde{B}_{j, i}\right)\right) u_{C}^{n, i}+\Delta t \sum_{j=i+1}^{I_{h}-1} A_{j} B_{i, j} u_{C}^{n, j} \Delta x_{j},
$$

for $i=1, \ldots, I_{h}-2$. The cases $i=0$ and $i=I_{h}-1$ can be handled similarly to obtain the same result, where the empty sums are taken as 0 .

From this it is clear that if each of the approximations $u_{C}^{n, i}$ is nonnegative, and provided $\Delta t$ is sufficiently small such that the term within the outer brackets is nonnegative, then each of the approximations $u_{C}^{n+1, i}$, for the subsequent time step, will also be nonnegative. Hence provided condition (4.1) is satisfied, then the approximations $u_{C}^{n+1, i}$ will be nonnegative. In the case that the denominator from (4.1) is zero for some $i$, such that the bound (4.1) is undefined, then $u_{C}^{n+1, i}$ can be seen from (4.4) to automatically satisfy the nonnegativity requirement, for any value of $\Delta t$.

Turning to the approximation for the discrete regime, it is immediately clear from the form of (3.5) that if all of the values $u_{C}^{n, i}$ and $u_{D}^{n, i}$ are nonnegative, then each $u_{D}^{n+1, i}$ will be nonnegative if for each $i=1, \ldots, N$ we have that $1-\Delta t a_{i}$ is nonnegative, giving rise to the condition (4.2).

Therefore if we choose a $\Delta t$ small enough that both (4.1) and (4.2) are satisfied, then our approximate solutions will remain nonnegative.

From now on we shall assume that conditions (4.1) and (4.2) are satisfied and that $c_{0}(x) \geq 0$ and each $d_{0, i} \geq 0$ so that our approximations remain nonnegative.

Remark 4.2. The bound (4.1) is dependent on the mesh and it is perhaps not immediately apparent how this bounding value might vary as we refine the mesh. In particular, it would be advantageous to confirm that it is indeed possible to find a constant $k_{1}$, such that conditions (3.1) and (4.1) can be satisfied simultaneously, whilst $h \searrow 0$. In the upcoming analysis we will place restrictions on the functions $a$ and $b$; these constraints will allow us to guarantee the existence of such a $k_{1}$.

The upcoming Theorem 5.3 imposes the restriction $a, b \in L_{\infty}$ on the restricted domains $[N, R]$ and $[N, R] \times$ $[N, R]$ respectively, allowing us to define the values $\alpha(R)$ and $\beta(R)$ as follows:

$$
\alpha(R)=\underset{x \in[N, R]}{\operatorname{ess} \sup } a(x) \text { and } \beta(R)=\operatorname{essup}_{(x, y) \in[N, R]^{2}} b(x \mid y) .
$$

This being the case, we have $A_{i} \leq \alpha(R)$ and $B_{k, i} \leq \beta(R)$ for all values of $i$ and $k$ admissible in (4.1). Furthermore, from (1.3) we may deduce that each $b_{i}(y) \leq y$, hence $\tilde{B}_{j, i} \leq R$. Finally, all mesh midpoints $x_{i}$ must clearly 
satisfy $x_{i} \geq N \geq 1>h$. Taken together, these bounds lead, via a simple calculation, to

$$
\frac{h}{\alpha(R)(\beta(R) R(R-N)+R N(N+1) / 2)} \leq \frac{x_{i}}{A_{i}\left(\sum_{k=0}^{i-1} x_{k} B_{k, i} \Delta x_{k}+\sum_{j=1}^{N} j \tilde{B}_{j, i}\right)},
$$

for $i=0,1, \ldots, I_{h}-1$. Hence, we have established a possible value for $k_{1}$, which ensures that (3.1) and (4.1) can be satisfied simultaneously as $h \searrow 0$.

\subsection{Mass conservation by the numerical solutions}

In Lemma 6.2 of [5], the exact solution to our system of equations (1.1) and (1.2) was shown to conserve mass between the two regimes. We now show that this property is shared by our numerical solutions.

Lemma 4.3. The approximate solutions generated by (3.3) and (3.5) conserve mass.

Proof. The mass associated with the approximate continuous regime solution, $u_{C}^{h}(x, t)$, is given by

$$
\begin{aligned}
\left\|u_{C}^{h}(\cdot, t)\right\|_{L_{1}^{1}(N, R)} & =\int_{N}^{R} \sum_{n=0}^{M-1} \sum_{i=0}^{I_{h}-1} \chi_{\Lambda_{i}}(x) \chi_{\tau_{n}}(t) u_{C}^{n, i} x \mathrm{~d} x \\
& =\sum_{n=0}^{M-1} \chi_{\tau_{n}}(t) \sum_{i=0}^{I_{h}-1} u_{C}^{n, i} \int_{N}^{R} \chi_{\Lambda_{i}}(x) x \mathrm{~d} x \\
& =\sum_{n=0}^{M-1} \chi_{\tau_{n}}(t) \sum_{i=0}^{I_{h}-1} x_{i} \Delta x_{i} u_{C}^{n, i}
\end{aligned}
$$

whilst the approximate solution $u_{D}^{h}(t)$ has associated mass given by

$$
\left\|u_{D}^{h}(t)\right\|_{X_{D}}=\sum_{i=1}^{N} i u_{D i}^{h}(t)=\sum_{i=1}^{N} i \sum_{n=0}^{M-1} \chi_{\tau_{n}}(t) u_{D}^{n, i}=\sum_{n=0}^{M-1} \chi_{\tau_{n}}(t) \sum_{i=1}^{N} i u_{D}^{n, i},
$$

where $X_{D}$ denotes the space $\mathbb{R}^{N}$ equipped with the weighted norm $\|v\|_{X_{D}}=\sum_{j=1}^{N} j\left|v_{j}\right|$, for $v=\left(v_{1}, v_{2}, \ldots, v_{N}\right)$. Summing expressions (4.6) and (4.7) gives the total mass:

$$
M^{h}(t)=\sum_{n=0}^{M-1} \chi_{\tau_{n}}(t)\left(\sum_{i=0}^{I_{h}-1} x_{i} \Delta x_{i} u_{C}^{n, i}+\sum_{i=1}^{N} i u_{D}^{n, i}\right) .
$$

First let us examine the mass accounted for by the continuous regime. From the relation (3.3) we get

$$
\begin{aligned}
\sum_{i=0}^{I_{h}-1} x_{i} u_{C}^{n+1, i} \Delta x_{i} & =\sum_{i=0}^{I_{h}-1} x_{i}\left(u_{C}^{n, i}+\frac{\Delta t}{x_{i} \Delta x_{i}}\left(F_{i+1 / 2}^{n}-F_{i-1 / 2}^{n}\right)-\frac{\Delta t}{x_{i}} S_{i}^{n}\right) \Delta x_{i} \\
& =\sum_{i=0}^{I_{h}-1} x_{i} u_{C}^{n, i} \Delta x_{i}+\Delta t \sum_{i=0}^{I_{h}-1}\left(F_{i+1 / 2}^{n}-F_{i-1 / 2}^{n}\right)-\Delta t \sum_{i=0}^{I_{h}-1} S_{i}^{n} \Delta x_{i} \\
& =\sum_{i=0}^{I_{h}-1} x_{i} u_{C}^{n, i} \Delta x_{i}-\Delta t \sum_{i=0}^{I_{h}-1} S_{i}^{n} \Delta x_{i} .
\end{aligned}
$$


The middle summation term is lost in going to the final line as the sum is telescoping with zero end terms. Now we consider the discrete regime mass; the generating relation (3.5) gives us

$$
\begin{aligned}
\sum_{i=1}^{N} i u_{D}^{n+1, i} & =\sum_{i=1}^{N} i\left(\left(1-\Delta t a_{i}\right) u_{D}^{n, i}+\Delta t \sum_{j=i+1}^{N} a_{j} b_{i, j} u_{D}^{n, j}+\Delta t \sum_{j=0}^{I_{h}-1} A_{j} \tilde{B}_{i, j} u_{C}^{n, j} \Delta x_{j}\right) \\
& =\sum_{i=1}^{N} i u_{D}^{n, i}-\Delta t \sum_{i=1}^{N} i a_{i} u_{D}^{n, i}+\Delta t \sum_{i=1}^{N} i \sum_{j=i+1}^{N} a_{j} b_{i, j} u_{D}^{n, j}+\Delta t \sum_{i=1}^{N} i \sum_{j=0}^{I_{h}-1} A_{j} \tilde{B}_{i, j} u_{C}^{n, j} \Delta x_{j} \\
& =\sum_{i=1}^{N} i u_{D}^{n, i}-\Delta t \sum_{i=2}^{N} i a_{i} u_{D}^{n, i}+\Delta t \sum_{j=2}^{N} a_{j} u_{D}^{n, j}\left(\sum_{i=1}^{j-1} i b_{i, j}\right)+\Delta t \sum_{j=0}^{I_{h}-1} \Delta x_{j}\left(A_{j} u_{C}^{n, j} \sum_{i=1}^{N} i \tilde{B}_{i, j}\right) \\
& =\sum_{i=1}^{N} i u_{D}^{n, i}+\Delta t \sum_{j=0}^{I_{h}-1} \Delta x_{j} S_{j}^{n} .
\end{aligned}
$$

The middle two terms cancel due to the mass conservation condition (1.4). Combining equations (4.9) and (4.10) we obtain

$$
\begin{aligned}
\sum_{i=0}^{I_{h}-1} x_{i} u_{C}^{n+1, i} \Delta x_{i}+\sum_{i=1}^{N} i u_{D}^{n+1, i} & =\sum_{i=0}^{I_{h}-1} x_{i} u_{C}^{n, i} \Delta x_{i}-\Delta t \sum_{i=0}^{I_{h}-1} S_{i}^{n} \Delta x_{i}+\sum_{i=1}^{N} i u_{D}^{n, i}+\Delta t \sum_{j=0}^{I_{h}-1} \Delta x_{j} S_{j}^{n} \\
& =\sum_{i=0}^{I_{h}-1} x_{i} u_{C}^{n, i} \Delta x_{i}+\sum_{i=1}^{N} i u_{D}^{n, i} .
\end{aligned}
$$

From repeated application of this equality it is easily seen that the bracketed expression appearing in (4.8) is equal for all values of $n$, and hence the total mass $M^{h}(t)$ remains constant.

\section{Convergence of the SCheme to A WeAK SOlution AS $h \rightarrow 0$}

Having determined the nonnegativity and mass conservative properties of the approximate solutions provided by (3.4) and (3.6), in this section we set out to prove that they converge, in some sense, to a limit as the parameter $h$, and by necessity $\Delta t$, go to zero, and show that this limit itself is an "exact" solution to our truncated model.

\subsection{Continuous fragmentation regime: convergence}

Let us start with the continuous regime approximations $\left\{u_{C}^{h}\right\}$. In order to prove the (weak) convergence of this family, we employ a weak compactness argument, utilising the Dunford-Pettis theorem (Thm. 2.5), which provides necessary and sufficient conditions for weak compactness in an $L_{1}$ space. We begin by proving the equiboundedness of the set $\left\{u_{C}^{h}\right\}$.

Lemma 5.1. The family of approximations $\left\{u_{C}^{h}\right\}$ is equibounded (uniformly bounded) in the space $L_{1}((N, R) \times$ $[0, T), x \mathrm{~d} x \mathrm{~d} t)$.

Proof. Recalling equation (4.6), we have for any $t \in[0, T)$ that

$$
\left\|u_{C}^{h}(\cdot, t)\right\|_{L_{1}^{1}(N, R)}=\sum_{n=0}^{M-1} \chi_{\tau_{n}}(t) \sum_{i=0}^{I_{h}-1} x_{i} \Delta x_{i} u_{C}^{n, i} .
$$

From the analysis of Lemma 4.1, each of the values $u_{C}^{n, i}$ is nonnegative, and as such the values $S_{i}^{n}$ are nonnegative. Therefore, from the last line of equation (4.9) we deduce that

$$
\sum_{i=0}^{I_{h}-1} x_{i} \Delta x_{i} u_{C}^{n, i} \leq \sum_{i=0}^{I_{h}-1} x_{i} \Delta x_{i} u_{C}^{n-1, i} \quad \text { for } n=1, \ldots, M-1 .
$$


Repeated application of this inequality yields

$$
\sum_{i=0}^{I_{h}-1} x_{i} \Delta x_{i} u_{C}^{n, i} \leq \sum_{i=0}^{I_{h}-1} x_{i} \Delta x_{i} u_{C}^{0, i}=\sum_{i=0}^{I_{h}-1} x_{i} \int_{\Lambda_{i}} c_{0}(x) \mathrm{d} x \leq \sum_{i=0}^{I_{h}-1} \frac{x_{i}}{x_{i-1 / 2}} \int_{\Lambda_{i}} c_{0}(x) x \mathrm{~d} x .
$$

The quantity $\frac{x_{i}}{x_{i-1 / 2}}$ can be bounded as follows:

$$
\frac{x_{i}}{x_{i-1 / 2}}=\frac{x_{i-1 / 2}+\frac{1}{2} \Delta x_{i}}{x_{i-1 / 2}} \leq 1+\frac{h}{2 N} \leq \frac{3}{2}
$$

We note this bound as it will appear regularly in subsequent calculations. Substituting this within (5.1) yields

$$
\sum_{i=0}^{I_{h}-1} x_{i} \Delta x_{i} u_{C}^{n, i} \leq \frac{3}{2}\left\|c_{0}\right\|_{L_{1}^{1}(N, R)},
$$

for $n=0, \ldots, M-1$. Replacing this inequality in our calculation gives us the following, which holds for all $t \in[0, T):$

$$
\left\|u_{C}^{h}(\cdot, t)\right\|_{L_{1}^{1}(N, R)} \leq \sum_{n=0}^{M-1} \chi_{\tau_{n}}(t) \frac{3}{2}\left\|c_{0}\right\|_{L_{1}^{1}(N, R)}=\frac{3}{2}\left\|c_{0}\right\|_{L_{1}^{1}(N, R)} .
$$

Integrating this inequality with respect to $t$ from 0 to $T$ we obtain the required equiboundedness of $\left\{u_{C}^{h}\right\}$ in the space $L_{1}((N, R) \times[0, T), x \mathrm{~d} x \mathrm{~d} t)$.

We now move on to prove the second of the two required conditions for the Dunford-Pettis theorem, namely equiintegrability. However, prior to this we collect all the conditions so far imposed on our model via the functions $a, b$ and $b_{i}$, the values $b_{j, i}$, and the initial distributions $c_{0}$ and $d_{0}$, and also on our mesh via the parameters $h$ and $\Delta t$.

Remark 5.2. Throughout the remaining analysis, the following conditions shall be assumed to be satisfied.

(1) The initial mass distributions within the continuous and discrete regimes are nonnegative, that is $c_{0}(x) \geq 0$ for all $x>N$ and $d_{0, i} \geq 0$ for $i=1, \ldots, N$.

(2) The functions $a$ and $b$ are nonnegative and belong to the spaces $L_{\infty, \text { loc }}([N, \infty))$ and $L_{\infty, \text { loc }}([N, \infty) \times[N, \infty))$, respectively.

(3) The functions $b$ and $b_{i}$, and the values $b_{j, i}$ are assumed to satisfy the conditions (1.3) and (1.4).

(4) Each of the functions $b_{i}$ is assumed to be nonnegative. This nonnegativity in conjunction with condition 2 and (1.3) is sufficient to guarantee that $b_{i} \in L_{\infty, \text { loc }}([N, \infty))$.

(5) There exist positive constants $k_{1}$ and $k_{2}$ such that the mesh parameters $h$ and $\Delta t$ satisfy

$$
k_{1} h \leq \Delta t \leq k_{2} h
$$

(6) To ensure that the approximate solutions remain nonnegative, the time step $\Delta t$ is assumed to satisfy the following constraints:

$$
0<\Delta t \leq \frac{x_{i}}{A_{i}\left(\sum_{k=0}^{i-1} x_{k} B_{k, i} \Delta x_{k}+\sum_{j=1}^{N} j \tilde{B}_{j, i}\right)} \text { for } i=0,1, \ldots, I_{h}-1,
$$

for all cases of the denominator being nonzero, and

$$
0<\Delta t \leq \frac{1}{a_{i}} \text { for } i=2, \ldots, N \text { such that } a_{i} \neq 0 .
$$


(7) There exists a constant $\theta>0$ such that

$$
K(R) \Delta t \leq \theta<1,
$$

where $K(R)=\alpha(R) \beta(R) R$, with $\alpha(R)$ and $\beta(R)$ as given in (4.5). This final condition shall be utilised in Theorem 5.3, which immediately follows.

Theorem 5.3. Under the assumptions outlined in Remark 5.2, the family $\left\{u_{C}^{h}\right\}$ is equiintegrable in $L_{1}((N, R) \times$ $[0, T), x \mathrm{~d} x \mathrm{~d} t)$.

Proof. Consider the constant sequence comprising solely of the initial data $c_{0} \in L_{1}((N, R), x \mathrm{~d} x)$. Clearly this sequence is convergent, therefore $\left\{c_{0}\right\}$ forms a weakly sequentially compact set in $L_{1}((N, R), x \mathrm{~d} x)$. Hence by the de la Vallee Poussin theorem (Thm. 2.3) there exists a nonnegative, convex function $\Phi \in C^{\infty}([0, \infty))$, with $\Phi(0)=0$ and $\Phi^{\prime}(0)=1$ such that $\Phi^{\prime}$ is concave, and $\Phi$ satisfies

$$
\frac{\Phi(x)}{x} \rightarrow \infty \text { as } x \rightarrow \infty \text { and } \int_{N}^{R} \Phi\left(c_{0}\right)(x) x \mathrm{~d} x<\infty
$$

The inequality (2.1) gives us

$$
\Phi\left(u_{C}^{n+1, i}\right)-\Phi\left(u_{C}^{n, i}\right) \leq\left(u_{C}^{n+1, i}-u_{C}^{n, i}\right) \Phi^{\prime}\left(u_{C}^{n+1, i}\right) .
$$

Multiplying this by $x_{i} \Delta x_{i}$ and summing over all $i$ gives

$$
\sum_{i=0}^{I_{h}-1} x_{i} \Delta x_{i}\left(\Phi\left(u_{C}^{n+1, i}\right)-\Phi\left(u_{C}^{n, i}\right)\right) \leq \sum_{i=0}^{I_{h}-1} x_{i} \Delta x_{i}\left(\left(u_{C}^{n+1, i}-u_{C}^{n, i}\right) \Phi^{\prime}\left(u_{C}^{n+1, i}\right)\right) .
$$

Utilising equation (3.3) we can rewrite this as

$$
\sum_{i=0}^{I_{h}-1} x_{i} \Delta x_{i}\left(\Phi\left(u_{C}^{n+1, i}\right)-\Phi\left(u_{C}^{n, i}\right)\right) \leq \sum_{i=0}^{I_{h}-1} x_{i} \Delta x_{i}\left(\frac{\Delta t}{x_{i} \Delta x_{i}}\left(F_{i+1 / 2}^{n}-F_{i-1 / 2}^{n}\right)-\frac{\Delta t}{x_{i}} S_{i}^{n}\right) \Phi^{\prime}\left(u_{C}^{n+1, i}\right) .
$$

Recalling the definition of $S_{i}^{n}$ from (3.2), we see that it must be nonnegative. Additionally, Lemma 2.4(ii) and Lemma 4.1 give $\Phi^{\prime}\left(u_{C}^{n+1, i}\right) \geq 0$, hence we can drop the term involving $S_{i}^{n}$ from $(5.2)$ and the inequality will still remain valid, giving us

$$
\sum_{i=0}^{I_{h}-1} x_{i} \Delta x_{i}\left(\Phi\left(u_{C}^{n+1, i}\right)-\Phi\left(u_{C}^{n, i}\right)\right) \leq \sum_{i=0}^{I_{h}-1} \Delta t\left(F_{i+1 / 2}^{n}-F_{i-1 / 2}^{n}\right) \Phi^{\prime}\left(u_{C}^{n+1, i}\right) .
$$

Omitting the last negative term from equation (4.3) yields the inequality

$$
F_{i+1 / 2}^{n}-F_{i-1 / 2}^{n} \leq x_{i} \Delta x_{i} \sum_{j=i+1}^{I_{h}-1} A_{j} B_{i, j} u_{C}^{n, j} \Delta x_{j}
$$

which, if placed in the previous inequality, results in

$$
\sum_{i=0}^{I_{h}-1} x_{i} \Delta x_{i}\left(\Phi\left(u_{C}^{n+1, i}\right)-\Phi\left(u_{C}^{n, i}\right)\right) \leq \Delta t \sum_{i=0}^{I_{h}-1} \sum_{j=i+1}^{I_{h}-1} x_{i} A_{j} B_{i, j} u_{C}^{n, j} \Delta x_{i} \Delta x_{j} \Phi^{\prime}\left(u_{C}^{n+1, i}\right) .
$$


Utilising Lemma 2.4(i) with $x=u_{C}^{n, j}$ and $y=u_{C}^{n+1, i}$ and noting that the constants $\alpha(R)$ and $\beta(R)$ bound the average values $A_{j}$ and $B_{i, j}$, we get

$$
\begin{aligned}
& \sum_{i=0}^{I_{h}-1} x_{i} \Delta x_{i}\left(\Phi\left(u_{C}^{n+1, i}\right)-\Phi\left(u_{C}^{n, i}\right)\right) \leq \alpha(R) \beta(R) \Delta t \sum_{i=0}^{I_{h}-1} \sum_{j=i+1}^{I_{h}-1} x_{i} \Delta x_{i} \Delta x_{j} u_{C}^{n, j} \Phi^{\prime}\left(u_{C}^{n+1, i}\right) \\
& \quad \leq \alpha(R) \beta(R) \Delta t\left(\sum_{i=0}^{I_{h}-1}\left(x_{i} \Delta x_{i} \Phi\left(u_{C}^{n+1, i}\right) \sum_{j=i+1}^{I_{h}-1} \Delta x_{j}\right)+\sum_{i=0}^{I_{h}-1}\left(x_{i} \Delta x_{i} \sum_{j=i+1}^{I_{h}-1} \Delta x_{j} \Phi\left(u_{C}^{n, j}\right)\right)\right) .
\end{aligned}
$$

As $j$ is restricted to be greater than $i$ we have $x_{j}>x_{i}$ for admissible $j$ and $i$. This allows us to switch $x_{i}$ for $x_{j}$ in the second term and take this within the inner summation. Following this we expand the summation over $j$ to give

$$
\begin{aligned}
& \sum_{i=0}^{I_{h}-1} x_{i} \Delta x_{i}\left(\Phi\left(u_{C}^{n+1, i}\right)-\Phi\left(u_{C}^{n, i}\right)\right) \\
& \quad \leq \alpha(R) \beta(R) \Delta t\left(\sum_{i=0}^{I_{h}-1}\left(x_{i} \Delta x_{i} \Phi\left(u_{C}^{n+1, i}\right) \sum_{j=i+1}^{I_{h}-1} \Delta x_{j}\right)+\sum_{i=0}^{I_{h}-1}\left(\Delta x_{i} \sum_{j=i+1}^{I_{h}-1} x_{j} \Delta x_{j} \Phi\left(u_{C}^{n, j}\right)\right)\right) \\
& \quad \leq \underbrace{\alpha(R) \beta(R) R}_{=K(R)} \Delta t\left(\sum_{i=0}^{I_{h}-1} x_{i} \Delta x_{i} \Phi\left(u_{C}^{n+1, i}\right)+\sum_{j=0}^{I_{h}-1} x_{j} \Delta x_{j} \Phi\left(u_{C}^{n, j}\right)\right)
\end{aligned}
$$

If we change the index variable from $j$ to $i$ in the second summation and re-arrange then we obtain

$$
(1-K(R) \Delta t) \sum_{i=0}^{I_{h}-1} x_{i} \Delta x_{i} \Phi\left(u_{C}^{n+1, i}\right) \leq(1+K(R) \Delta t) \sum_{i=0}^{I_{h}-1} x_{i} \Delta x_{i} \Phi\left(u_{C}^{n, i}\right) .
$$

By the final assumption of Remark 5.2, we have $1-K(R) \Delta t>0$ allowing us to divide through to get

$$
\sum_{i=0}^{I_{h}-1} x_{i} \Delta x_{i} \Phi\left(u_{C}^{n+1, i}\right) \leq\left(1+\frac{2 K(R) \Delta t}{1-K(R) \Delta t}\right) \sum_{i=0}^{I_{h}-1} x_{i} \Delta x_{i} \Phi\left(u_{C}^{n, i}\right) .
$$

Repeated application of this inequality yields

$$
\begin{aligned}
\sum_{i=0}^{I_{h}-1} x_{i} \Delta x_{i} \Phi\left(u_{C}^{n+1, i}\right) & \leq\left(1+\frac{2 K(R) \Delta t}{1-K(R) \Delta t}\right)^{n+1} \sum_{i=0}^{I_{h}-1} x_{i} \Delta x_{i} \Phi\left(u_{C}^{0, i}\right) \\
& \leq \exp \left(\frac{2 K(R) \Delta t(n+1)}{1-K(R) \Delta t}\right) \sum_{i=0}^{I_{h}-1} x_{i} \Delta x_{i} \Phi\left(u_{C}^{0, i}\right) .
\end{aligned}
$$

For values of $t$ in the interval $\tau_{n}=\left[t_{n}, t_{n+1}\right)$ this gives us

$$
\begin{aligned}
\int_{N}^{R} \Phi\left(u_{C}^{h}(x, t)\right) x \mathrm{~d} x & =\sum_{i=0}^{I_{h}-1} x_{i} \Delta x_{i} \Phi\left(u_{C}^{n, i}\right) \leq \exp \left(\frac{2 K(R) t}{1-K(R) \Delta t}\right) \sum_{i=0}^{I_{h}-1} x_{i} \Delta x_{i} \Phi\left(u_{C}^{0, i}\right) \\
& =\exp \left(\frac{2 K(R) t}{1-K(R) \Delta t}\right) \sum_{i=0}^{I_{h}-1} x_{i} \Delta x_{i} \Phi\left(\frac{1}{\Delta x_{i}} \int_{\Lambda_{i}} c_{0}(x) \mathrm{d} x\right) .
\end{aligned}
$$


An application of Jensen's inequality ([40], Thm. 2.2) allows us to switch the order of $\Phi$ and integration to get

$$
\begin{aligned}
\int_{N}^{R} \Phi\left(u_{C}^{h}(x, t)\right) x \mathrm{~d} x & \leq \exp \left(\frac{2 K(R) t}{1-K(R) \Delta t}\right) \sum_{i=0}^{I_{h}-1} x_{i} \int_{\Lambda_{i}} \Phi\left(c_{0}(x)\right) \mathrm{d} x \\
& \leq \frac{3}{2} \exp \left(\frac{2 K(R) t}{1-K(R) \Delta t}\right) \sum_{i=0}^{I_{h}-1} \int_{\Lambda_{i}} \Phi\left(c_{0}(x)\right) x \mathrm{~d} x .
\end{aligned}
$$

By assumption 6 of Remark 5.2, that $K(R) \Delta t \leq \theta<1$, we deduce that

$$
\int_{N}^{R} \Phi\left(u_{C}^{h}(x, t)\right) x \mathrm{~d} x \leq \frac{3}{2} \exp \left(\frac{2 K(R) t}{1-\theta}\right) \int_{N}^{R} \Phi\left(c_{0}(x)\right) x \mathrm{~d} x,
$$

which holds for all $t \in[0, T)$. Integrating the inequality with respect to $t$ from 0 to $T$ confirms the equiintegrability of the family $\left\{u_{C}^{h}\right\}$ in the space $L_{1}((N, R) \times[0, T), x \mathrm{~d} x \mathrm{~d} t)$.

By Theorem 2.5 (Dunford-Pettis theorem), the sequence $\left\{u_{C}^{h}\right\}$ forms a weakly sequentially compact set in the space $L_{1}((N, R) \times[0, T), x \mathrm{~d} x \mathrm{~d} t)$. This implies the existence of a subsequence $\left\{u_{C}^{h j}\right\}$ and a function $u_{C}^{R} \in L_{1}((N, R) \times[0, T), x \mathrm{~d} x \mathrm{~d} t)$ such that $u_{C}^{h j} \rightarrow u_{C}^{R}$ in $L_{1}((N, R) \times[0, T), x \mathrm{~d} x \mathrm{~d} t)$ as $j \rightarrow \infty$ and $h_{j} \rightarrow 0$.

Remark 5.4. From now on this convergent subsequence will be considered implicitly, unless otherwise stated; as such we now use the notation $\left\{u_{C}^{h}\right\}$ to denote such a convergent subsequence, the choice of which, we note, may not be unique.

\subsection{Continuous fragmentation regime: weak solution}

Having shown that our sequence of approximations converges (weakly) to a limit, we now aim to show that this limit provides a solution to our truncated equation (1.6). Precisely, we intend to show that the function $u_{C}^{R}$ satisfies the following criterion.

Definition 5.5. The function $u_{C}^{R}$ is a weak solution of equation (1.6), if it satisfies

$$
\begin{aligned}
\int_{0}^{T} & \int_{N}^{R} x u_{C}^{R}(x, t) \frac{\partial \varphi}{\partial t}(x, t) \mathrm{d} x \mathrm{~d} t+\int_{N}^{R} x c_{0}(x) \varphi(x, 0) \mathrm{d} x \\
& =\int_{0}^{T} \int_{N}^{R} \mathcal{F}^{R}\left(x u_{C}^{R}\right)(x, t) \frac{\partial \varphi}{\partial x}(x, t) \mathrm{d} x \mathrm{~d} t+\int_{0}^{T} \int_{N}^{R} S\left(x u_{C}^{R}\right)(x, t) \varphi(x, t) \mathrm{d} x \mathrm{~d} t
\end{aligned}
$$

for all functions $\varphi$, which are twice continuously differentiable functions on $(N, R) \times(0, T)$, such that $\varphi$ and each of its derivatives up to order 2 may be continuously extended to $[N, R] \times[0, T)$, and such that for each fixed $x \in[N, R]$, the support of $\varphi$ with respect to $t$ is a compact subset of $[0, T)$. We denote the set of such extended functions by $C_{c}^{2}([N, R] \times[0, T))$. We note that the weak formulation (5.3) was obtained from (1.6) in the usual manner, recalling the zero boundary conditions established in Lemma 1.1. Finally, recalling Remark 1.2 would allow us to alternatively re-write (5.3) without the requirement for an $x$-derivative on the right-hand side.

Definition 5.6. In the analysis which follows we make use of the following three approximations to $x$ over the domain $(N, R)$. First we have the left endpoint approximation, defined by

$$
\xi^{h}: x \in(N, R) \rightarrow \xi^{h}(x)=\sum_{i=0}^{I_{h}-1} \chi_{\Lambda_{i}}(x) x_{i-1 / 2} .
$$


Secondly we consider the midpoint approximation, defined by

$$
X^{h}: x \in(N, R) \rightarrow X^{h}(x)=\sum_{i=0}^{I_{h}-1} \chi_{\Lambda_{i}}(x) x_{i},
$$

and finally we introduce the right endpoint approximation given by

$$
\Xi^{h}: x \in(N, R) \rightarrow \Xi^{h}(x)=\sum_{i=0}^{I_{h}-1} \chi_{\Lambda_{i}}(x) x_{i+1 / 2} .
$$

Remark 5.7. It is a simple exercise to show that the three approximations, introduced above, converge pointwise (uniformly) to $x$ over the domain $(N, R)$ as the mesh parameter $h$ goes to 0 . The reader may find details given in Lemma 5.2 .3 of [4].

We are now in a position to proceed with our proof that $u_{C}^{R}$ is a weak solution to (1.6).

Definition 5.8. Let $\varphi \in C_{c}^{2}([N, R] \times[0, T))$; then for sufficiently small $\Delta t$, the support of $\varphi$ with respect to $t$ lies within $\left[0, t_{M-1}\right]$. We define $\varphi_{i}^{n}$ as an approximation of $\varphi$ on $\Lambda_{i} \times \tau_{n}$ by

$$
\varphi_{i}^{n}=\frac{1}{\Delta t} \int_{\tau_{n}} \varphi\left(x_{i-1 / 2}, t\right) \mathrm{d} t
$$

with $\varphi_{i}^{M-1}=\varphi_{i}^{M}=0$ for admissible $i$.

Rearranging equation (3.3), multiplying by $\varphi_{i}^{n}$ and summing over $n=0, \ldots, M-1$ and $i=0, \ldots, I_{h}-1$, gives us

$$
\sum_{n=0}^{M-1} \sum_{i=0}^{I_{h}-1}\left(x_{i} \Delta x_{i}\left(u_{C}^{n+1, i}-u_{C}^{n, i}\right) \varphi_{i}^{n}-\Delta t\left(F_{i+1 / 2}^{n}-F_{i-1 / 2}^{n}\right) \varphi_{i}^{n}+\Delta t \Delta x_{i} S_{i}^{n} \varphi_{i}^{n}\right)=0 .
$$

Rearrangement of the summations and utilising the compact support of $\varphi$ and the zero boundary flux gives us the following equality:

$$
\begin{aligned}
\sum_{n=0}^{M-1} & \sum_{i=0}^{I_{h}-1} x_{i} \Delta x_{i} u_{C}^{n+1, i}\left(\varphi_{i}^{n+1}-\varphi_{i}^{n}\right)+\sum_{i=0}^{I_{h}-1} x_{i} \Delta x_{i} u_{C}^{0, i} \varphi_{i}^{0} \\
& -\sum_{n=0}^{M-1} \sum_{i=0}^{I_{h}-1} \Delta t F_{i+1 / 2}^{n}\left(\varphi_{i+1}^{n}-\varphi_{i}^{n}\right)-\sum_{n=0}^{M-1} \sum_{i=0}^{I_{h}-1} \Delta t \Delta x_{i} S_{i}^{n} \varphi_{i}^{n}=0 .
\end{aligned}
$$

The above equality can be seen as the discrete equivalent of the weak formulation (5.3). Our approach now involves taking the limit as $h \rightarrow 0$ of (5.4) and showing that we do indeed obtain (5.3) with $u_{C}^{R}$ as a weak solution. Observing the terms of (5.3) we see that the integrals are with respect to the measure $\mathrm{d} x \mathrm{~d} t$ whilst we have shown that convergence occurs in the space with weighted measure $x \mathrm{~d} x \mathrm{~d} t$. At this point we highlight the use of Theorem 2.1 to switch spaces but retain convergence.

Theorem 5.9. Under the assumptions outlined in Remark 5.2, the function $u_{C}^{R}$ obtained as the limit of the sequence $\left\{u_{C}^{h}\right\}$, is a weak solution of our equation, satisfying (5.3).

Proof. The first three terms of (5.4) correspond closely to those set out in equation (2.21) of [34], which were shown to converge to limits similar in form to the relevant terms from (5.3). For full details of convergence in the case of (5.4) and (5.3), the reader is directed towards ([4], Thm. 5.2.6). However, the arguments employed for our three terms mirror those employed in Theorem 2.3.1 of [34], and as such are excluded here. Therefore, 
we focus our attention on the final terms of (5.4) and (5.3), which account for the mass transfer from our continuous regime to the discrete regime. The fourth term from equation (5.4) is given fully by

$$
\begin{aligned}
\sum_{n=0}^{M-1} \sum_{i=0}^{I_{h}-1} A_{i}\left(\sum_{j=1}^{N} j \tilde{B}_{j, i}\right) u_{C}^{n, i} \varphi_{i}^{n} \Delta x_{i} \Delta t & =\sum_{n=0}^{M-1} \sum_{i=0}^{I_{h}-1} \int_{\tau_{n}} \int_{\Lambda_{i}} a^{h}(v)\left(\sum_{j=1}^{N} j b_{j}^{h}(v)\right) u_{C}^{h} \varphi\left(\xi^{h}(v), t\right) \mathrm{d} v \mathrm{~d} t \\
& =\int_{0}^{T} \int_{N}^{R} a^{h}(v)\left(\sum_{j=1}^{N} j b_{j}^{h}(v)\right) u_{C}^{h}(v, t) \varphi\left(\xi^{h}(v), t\right) \mathrm{d} v \mathrm{~d} t .
\end{aligned}
$$

The pointwise convergence of $a^{h}, b_{j}^{h}$ and $\xi^{h}$ along with the continuity of $\varphi$ means that

$$
a^{h}(v)\left(\sum_{j=1}^{N} j b_{j}^{h}(v)\right) \varphi\left(\xi^{h}(v), t\right) \rightarrow a(v)\left(\sum_{j=1}^{N} j b_{j}(v)\right) \varphi(v, t),
$$

for all $t \in[0, T)$ and almost all $v \in(R, N)$ as $h \rightarrow 0$. Since $a$ and $b_{i}$ are in $L_{\infty, \text { loc }}([N, \infty))$ and $\varphi$ is $C^{2}$ on $[N, R] \times[0, T)$ with compact support (hence is a bounded function), the expressions on either side belong to $L_{\infty}((N, R) \times[0, T))$, with the left-hand side being uniformly bounded w.r.t. $h$. Hence, with $u_{C}^{h} \rightarrow u_{C}^{R}$ in $L_{1}((N, R) \times[0, T), \mathrm{d} v \mathrm{~d} t)$, applying Theorem 2.2, yields

$$
\begin{aligned}
\int_{0}^{T} \int_{N}^{R} a^{h}(v)\left(\sum_{j=1}^{N} j b_{j}^{h}(v)\right) u_{C}^{h}(v, t) \varphi\left(\xi^{h}(v), t\right) \mathrm{d} v \mathrm{~d} t & \rightarrow \int_{0}^{T} \int_{N}^{R} a(v)\left(\sum_{j=1}^{N} j b_{j}(v)\right) u_{C}^{R}(v, t) \varphi(v, t) \mathrm{d} v \mathrm{~d} t \\
& =\int_{0}^{T} \int_{N}^{R} S\left(v u_{C}^{R}\right)(v, t) \varphi(v, t) \mathrm{d} v \mathrm{~d} t .
\end{aligned}
$$

Taken together, the convergence of the first three terms of (5.4), in addition to (5.5) show that $u_{C}^{R}$ satisfies (5.3) for all $\varphi \in C_{c}^{2}([N, R] \times[0, T))$, and hence $u_{C}^{R}$ is a weak solution, as set out in Definition 5.5.

\subsection{Discrete fragmentation regime: convergence}

Now let us consider the discrete regime approximations. This is treated by a similar approach to the one we adopted for the continuous regime equation, but as a first step we establish a bound on the values $u_{D}^{n, i}$.

Lemma 5.10. There exists a constant $C$, independent of $h$ and $R$, such that for all values of $n$ and $i$ we have

$$
0 \leq u_{D}^{n, i} \leq C
$$

Proof. The nonnegativity of $u_{D}^{n, i}$ follows from Lemma 4.1. We shall therefore concentrate on the upper bound. From Lemma 4.3 we have, for all admissible $n$, that the following holds:

$$
\begin{aligned}
\sum_{i=0}^{I_{h-1}} x_{i} \Delta x_{i} u_{C}^{n, i}+\sum_{i=1}^{N} i u_{D}^{n, i} & =\sum_{i=0}^{I_{h-1}} x_{i} \Delta x_{i} u_{C}^{0, i}+\sum_{i=1}^{N} i u_{D}^{0, i}=\sum_{i=0}^{I_{h-1}} x_{i} \int_{\Lambda_{i}} c_{0}(x) \mathrm{d} x+\sum_{i=1}^{N} i d_{0 i} \\
& \leq \frac{3}{2} \sum_{i=0}^{I_{h-1}} \int_{\Lambda_{i}} c_{0}(x) x \mathrm{~d} x+\sum_{i=1}^{N} i d_{0 i} \leq \frac{3}{2} \int_{N}^{\infty} c_{0}(x) x \mathrm{~d} x+\sum_{i=1}^{N} i d_{0 i}=C<\infty
\end{aligned}
$$

Therefore, for all $n$ and $i$ we have that

$$
u_{D}^{n, i} \leq C,
$$

where the constant $C$ is independent of the mesh parameter $h$ and the truncation parameter $R$. 
Theorem 5.11. For each $i=1, \ldots, N$, the family $\left\{u_{D i}^{h}\right\}$ forms a sequentially weakly compact set in $L_{1}([0, T))$, hence must have a weakly convergent subsequence.

Proof. By the bound obtained in Lemma 5.10 the family $\left\{u_{D i}^{h}\right\}$ is bounded in $L_{\infty}([0, T))$ and hence sequentially weakly compact in $L_{1}([0, T))$.

Remark 5.12. We note that what we seek is a collection of values $\left\{h^{j}\right\}$, such that all of the sequences $\left\{u_{D i}^{h^{j}}\right\}$, for $i=1, \ldots, N$, converge weakly, as $j \rightarrow \infty$ and $h_{j} \rightarrow 0$. Such a sequence $\left\{h^{j}\right\}$ may be obtained by means of a diagonal argument. From now on the convergent subsequences $\left\{u_{D i}^{h^{j}}\right\}$ are considered implicitly and we use $\left\{u_{D i}^{h}\right\}$ to denote said subsequences, unless otherwise stated. Let us denote the weak limit of $\left\{u_{D i}^{h}\right\}$ by $u_{D i}^{R}$ (note the upper case superscript notation for the limit).

\subsection{Discrete fragmentation regime: weak solution}

Having established the convergence of our sequence of approximations we now aim to determine whether the limit produced provides a solution to the equation (1.8) and if so in what sense. As such, following on from Definition 5.5, we introduce

Definition 5.13. We say that the function $u_{D i}^{R}$ is a weak solution of equation (1.8) if it satisfies

$$
\begin{aligned}
\int_{0}^{T} & u_{D i}^{R}(t) \frac{\mathrm{d} \phi}{\mathrm{d} t}(t) \mathrm{d} t+d_{0_{i}} \phi(0)-\int_{0}^{T} a_{i} u_{D i}^{R}(t) \phi(t) \mathrm{d} t \\
& +\int_{0}^{T} \sum_{j=i+1}^{N} a_{j} b_{i, j} u_{D j}^{R}(t) \phi(t) \mathrm{d} t+\int_{0}^{T} \int_{N}^{R} a(y) b_{i}(y) u_{C}^{R}(y, t) \phi(t) \mathrm{d} y \mathrm{~d} t=0
\end{aligned}
$$

for any $\phi \in C_{c}^{2}([0, T))$, where $C_{c}^{2}([0, T))$ is defined in an analogous fashion to $C_{c}^{2}([N, R] \times[0, T))$ from Definition 5.5.

Theorem 5.14. The functions $u_{D i}^{R}$ obtained as weak limits of the sequences $\left\{u_{D i}^{h}\right\}$ are indeed weak solutions of (1.8), satisfying equation (5.7) for any $\phi \in C_{c}^{2}([0, T))$.

Proof. For such a function $\phi$, let us denote its approximation over $\tau_{n}$ by $\phi^{n}$, which is defined as

$$
\phi^{n}=\frac{1}{\Delta t} \int_{\tau_{n}} \phi(t) \mathrm{d} t \text { for } n=0, \ldots, M-1,
$$

and $\phi^{M}=0$. Multiplying (3.5) by $\phi^{n}$ and summing over $n$ from 0 to $M-1$, gives us the following equality:

$$
\sum_{n=0}^{M-1}\left(u_{D}^{n+1, i}-u_{D}^{n, i}\right) \phi^{n}=-\sum_{n=0}^{M-1} a_{i} u_{D}^{n, i} \phi^{n} \Delta t+\sum_{n=0}^{M-1} \sum_{j=i+1}^{N} a_{j} b_{i, j} u_{D}^{n, j} \phi^{n} \Delta t+\sum_{n=0}^{M-1} \sum_{j=0}^{I_{h}-1} A_{j} \tilde{B}_{i, j} u_{C}^{n, j} \phi^{n} \Delta x_{j} \Delta t .
$$

Since $\phi$ is compactly supported, for sufficiently small $\Delta t$ we have $\phi^{M-1}=0$; then, further manipulation of the first term yields

$$
\begin{aligned}
& \sum_{n=0}^{M-1} u_{D}^{n+1, i}\left(\phi^{n+1}-\phi^{n}\right)+u_{D}^{0, i} \phi^{0}-\sum_{n=0}^{M-1} a_{i} u_{D}^{n, i} \phi^{n} \Delta t \\
& \quad+\sum_{n=0}^{M-1} \sum_{j=i+1}^{N} a_{j} b_{i, j} u_{D}^{n, j} \phi^{n} \Delta t+\sum_{n=0}^{M-1} \sum_{j=0}^{I_{h}-1} A_{j} \tilde{B}_{i, j} u_{C}^{n, j} \phi^{n} \Delta x_{j} \Delta t=0 .
\end{aligned}
$$


Looking more closely at the first term above we can rewrite it as

$$
\begin{aligned}
\sum_{n=0}^{M-1} u_{D}^{n+1, i}\left(\phi^{n+1}-\phi^{n}\right) & =\underbrace{u_{D}^{M, i}\left(\phi^{M}-\phi^{M-1}\right)}_{=0}+\sum_{n=0}^{M-2} \int_{\tau_{n+1}} u_{D i}^{h}(t) \frac{\phi(t)-\phi(t-\Delta t)}{\Delta t} \mathrm{~d} t \\
& =\int_{0}^{T} \chi_{[\Delta t, T)}(t) u_{D i}^{h}(t) \frac{\phi(t)-\phi(t-\Delta t)}{\Delta t} \mathrm{~d} t .
\end{aligned}
$$

Assuming that $t \in(0, T)$ and $\Delta t \leq t$, then a Taylor series expansion of $\phi(t-\Delta t)$ about $t$ gives

$$
\phi(t-\Delta t)=\phi(t)-\Delta t \frac{\mathrm{d} \phi}{\mathrm{d} t}(t)+O\left(\Delta t^{2}\right) .
$$

Therefore, we have

$$
\chi_{[\Delta t, T)}(t) \frac{\phi(t)-\phi(t-\Delta t)}{\Delta t}=\left\{\begin{array}{cc}
\frac{\Delta t \frac{\mathrm{d} \phi}{\mathrm{d} t}(x, t)+O\left(\Delta t^{2}\right)}{\Delta t}, & \Delta t \leq t, \\
0, & \Delta t>t .
\end{array}\right.
$$

As such, the left-hand side of (5.9) can be seen to converge pointwise to $\frac{\mathrm{d} \phi}{\mathrm{d} t}$ on $(0, T)$, as $h$, and by condition (3.1), $\Delta t$ goes to 0 . Since $\phi$ and its derivative $\frac{\mathrm{d} \phi}{\mathrm{d} t}$ must be bounded we can bound the left-hand side above, with the bound being uniform w.r.t $h$. Then, as $u_{D i}^{h} \rightarrow u_{D i}^{R}$ in $L_{1}([0, T))$, applying Theorem 2.2, as before, gives us

$$
\int_{0}^{T} \chi_{[\Delta t, T)}(t) u_{D i}^{h}(t) \frac{\phi(t)-\phi(t-\Delta t)}{\Delta t} \mathrm{~d} t \rightarrow \int_{0}^{T} u_{D i}^{R}(t) \frac{\mathrm{d} \phi}{\mathrm{d} t}(t) \mathrm{d} t .
$$

By definition, $u_{D}^{0, i}=d_{0 i}$, and since $\phi$ is $C^{2}$ with compact support, its derivative must be bounded, from which we deduce that

$$
\phi^{0}=\frac{1}{\Delta t} \int_{0}^{\Delta t} \phi(t) \mathrm{d} t \rightarrow \phi(0)
$$

as $h$ goes to 0 . Therefore

$$
u_{D}^{0, i} \phi^{0} \rightarrow d_{0 i} \phi(0) \text { as } h \rightarrow 0 .
$$

By temporarily defining $b_{i, i}$ to be -1 , we can combine the third and fourth terms of (5.8), writing them as

$$
\sum_{n=0}^{M-1} \sum_{j=i}^{N} a_{j} b_{i, j} u_{D}^{n, j} \phi^{n} \Delta t=\sum_{n=0}^{M-1} \sum_{j=i}^{N} \int_{\tau_{n}} a_{j} b_{i, j} u_{D j}^{h}(t) \phi(t) \mathrm{d} t=\int_{0}^{T} \sum_{j=i}^{N} a_{j} b_{i, j} u_{D j}^{h}(t) \phi(t) \mathrm{d} t,
$$

and since $u_{D j}^{h} \rightarrow u_{D j}^{R}$ in $L_{1}([0, T))$, for each $j$, we have

$$
\int_{0}^{T} \sum_{j=i}^{N} a_{j} b_{i, j} u_{D j}^{h}(t) \phi(t) \mathrm{d} t \rightarrow \int_{0}^{T} \sum_{j=i}^{N} a_{j} b_{i, j} u_{D j}^{R}(t) \phi(t) \mathrm{d} t=-\int_{0}^{T} a_{i} u_{D i}^{R}(t) \phi(t) \mathrm{d} t+\int_{0}^{T} \sum_{j=i+1}^{N} a_{j} b_{i, j} u_{D j}^{R}(t) \phi(t) \mathrm{d} t,
$$

giving us the third and fourth terms of our weak formulation (5.7). Rewriting the final term of our discrete relation, we get

$$
\begin{aligned}
\sum_{n=0}^{M-1} \sum_{j=0}^{I_{h}-1} A_{j} \tilde{B}_{i, j} u_{C}^{n, j} \phi^{n} \Delta x_{j} \Delta t & =\sum_{n=0}^{M-1} \sum_{j=0}^{I_{h}-1} \int_{\tau_{n}} \int_{\Lambda_{j}} a^{h}(y) b_{i}^{h}(y) \phi(t) u_{C}^{h}(y, t) \mathrm{d} y \mathrm{~d} t \\
& =\int_{0}^{T} \int_{N}^{R} a^{h}(y) b_{i}^{h}(y) \phi(t) u_{C}^{h}(y, t) \mathrm{d} y \mathrm{~d} t .
\end{aligned}
$$


From Remark 3.1 we have $a^{h}(y)$ and $b_{i}^{h}(y)$ converging pointwise to $a(y)$ and $b_{i}(y)$ respectively, and along with $\phi$ are bounded (uniformly with respect to $h$ ), a final application of Theorem 2.2 allows us to deduce that

$$
\int_{0}^{T} \int_{N}^{R} a^{h}(y) b_{i}^{h}(y) \phi(t) u_{C}^{h}(y, t) \mathrm{d} y \mathrm{~d} t \rightarrow \int_{0}^{T} \int_{N}^{R} a(y) b_{i}(y) u_{C}^{R}(y, t) \phi(t) \mathrm{d} y \mathrm{~d} t
$$

as the mesh size parameter $h \rightarrow 0$. Taking the results (5.10)-(5.13), we see that by letting $h \rightarrow 0$ in (5.8) we obtain the weak formulation (5.7), hence $u_{D i}^{R}$ is indeed a weak solution of (1.8).

In this section we established the weak convergence of a subsequence of our sequence of approximate solutions as the mesh parameter was decreased to zero. The limits were shown to provide a set of weak solutions to the truncated equations (1.6) and (1.8). However, there are a number of questions which remain unanswered which we seek to address in the following section.

\section{UNiqUENESS AND DIFFERENTIABILITY OF SOLUTIONS AND CONVERGENCE AS $R \rightarrow \infty$}

In the previous section, we formed approximate solutions to a truncated version of our system. A subsequence of these approximations was shown to converge to a weak solution to our problem, as the underlying mesh was refined. This convergence of subsequences, rather than the full sequence, raises the possibility of nonunique solutions, with each convergent subsequence possibly offering a different solution. In this section, by utilising the framework and theory of operator semigroups, we seek to address this, showing that any limits must coincide, providing a unique solution. Further, we would like to establish whether this solution may in fact display extra regularity, as we might expect from the results in [5]. The section is completed with an analysis of the truncation error introduced by restricting the continuous domain to $(N, R)$.

The theory and methods of operator semigroups have been widely utilised in the study of coagulationfragmentation problems, with the first such application by Aizenman and Bak [1], where the binary coagulationfragmentation equation was considered under the assumption of constant rate kernels. The approach adopted in [1] involved a sequence of truncated approximating problems, each of which was shown to give rise to a semigroup. The resulting sequence of semigroups was found to converge to a limit semigroup, which was then shown to provide a solution to the full problem. Using a similar approach, McLaughlin et al. [44] considered the multiple fragmentation equation, establishing the existence of solutions under more relaxed constraints on the fragmentation kernel. This was extended with the addition of a coagulation process in [43]. An alternative approach, involving substochastic semigroups and the Kato-Voigt perturbation theorem [54], was first applied to the field of fragmentation problems by Banasiak [6], who considered a particular class of power law fragmentation kernels. This approach was generalised for multiple fragmentation by Lamb [38] and Banasiak and Arlotti [8], who established the existence of unique mass-conserving positive solutions under suitable constraints on the fragmentation rate. This approach has proved particularly fruitful and has been applied to a wide range of coagulation-fragmentation problems, for example [7,9-15,17,41,52].

Following the approach of Chapter 8 from [8], we introduce the space $X_{C}^{R}=L_{1}((N, R), x \mathrm{~d} x)$ with the aim of recasting the equation (1.5) as an abstract Cauchy problem, as was carried out for (1.1) in [5]. Motivated by the terms appearing on the right-hand side of (1.5) we define the following linear operators $A_{C}^{R}$ and $B_{C}^{R}$ on the space $X_{C}^{R}$ :

$$
\left(A_{C}^{R} f\right)(x)=-a(x) f(x) \text { and }\left(B_{C}^{R} f\right)(x)=\int_{x}^{R} a(y) b(x \mid y) f(y) \mathrm{d} y \text { for } N<x<R,
$$

with the respective domains

$$
D\left(A_{C}^{R}\right)=\left\{f \in X_{C}^{R}: A_{C}^{R} f \in X_{C}^{R}\right\}, \quad D\left(B_{C}^{R}\right)=\left\{f \in X_{C}^{R}: B_{C}^{R} f \in X_{C}^{R}\right\} .
$$


Equation (1.5) is then recast as the following abstract Cauchy problem in the space $X_{C}^{R}$ :

$$
\frac{\mathrm{d}}{\mathrm{d} t} u_{C}^{R}(t)=\left(A_{C}^{R}+B_{C}^{R}\right)\left[u_{C}^{R}(t)\right], \quad t>0 ; \quad u_{C}^{R}(0)=c_{0}^{R}=\chi_{(N, R)}(x) c_{0}(x) .
$$

Here $u_{C}^{R}$ denotes an $X_{C}^{R}$-valued function rather than the scalar-valued function of two variables from the previous section. However due to the relationship between the spaces $L_{1}\left(I, L_{1}(\Omega, \mathrm{d} \mu)\right)$ and $L_{1}(\Omega \times I$, $\mathrm{d} \mu \mathrm{d} t)$, we may switch between the two, with each $L_{1}$-valued solution to (6.1) providing us with a scalar-valued solution to (1.5) and vice versa.

In order to similarly recast the discrete equations (1.8), we introduce the space $X_{D}=\mathbb{R}^{N}$, equipped with the weighted norm:

$$
\|v\|_{X_{D}}=\sum_{j=1}^{N} j\left|v_{j}\right|, \text { where } v=\left(v_{1}, \ldots, v_{N}\right) .
$$

The system (1.8) then becomes

$$
\frac{\mathrm{d}}{\mathrm{d} t} u_{D}^{R}(t)=\left(A_{D}+B_{D}\right)\left[u_{D}^{R}(t)\right]+C_{R}\left[u_{C}^{R}(t)\right], \quad t>0 ; \quad u_{D}^{R}(0)=d_{0},
$$

where $A_{D}$ and $B_{D}$ are defined on $X_{D}$, by

$$
\left(A_{D} v\right)_{i}=-a_{i} v_{i} \text { and }\left(B_{D} v\right)_{i}=\sum_{j=i+1}^{N} a_{j} b_{i, j} v_{j}, \text { for } i=1, \ldots, N,
$$

$u_{C}^{R}$ is the solution of (6.1) and where $C_{R}: D\left(C_{R}\right) \subseteq X_{C}^{R} \rightarrow X_{D}$ is given by

$$
\left(C_{R} f\right)_{i}=\int_{N}^{R} a(y) b_{i}(y) f(y) \mathrm{d} y, \quad D\left(C_{R}\right)=\left\{f \in X_{C}^{R}: C_{R} f \in X_{D}\right\} .
$$

Assuming that the functions $a, b$ and $b_{i}$ retain the properties imposed in Remark 5.2, then the following properties hold for the operators $A_{C}^{R}, B_{C}^{R}$ and $C_{R}$.

Lemma 6.1. The operators $A_{C}^{R}$ and $B_{C}^{R}$ are bounded linear operators on the space $X_{C}^{R}$, and $C_{R}$ is a bounded linear operator from $X_{C}^{R}$ to $X_{D}$. In addition, $\left\|B_{C}^{R} f\right\|_{X_{C}^{R}} \leq\left\|A_{C}^{R} f\right\|_{X_{C}^{R}}$ and $\left\|C_{R} f\right\|_{X_{D}} \leq\left\|A_{C}^{R} f\right\|_{X_{C}^{R}}$ for all $f \in X_{C}^{R}$, with the norms of the three operators each being bounded by $\alpha(R)$, where $\alpha(R)$ was given in (4.5).

Proof. The boundedness of $A_{C}^{R}$ is an immediate consequence of the local boundedness of the function $a$, in addition to the truncation of the continuous mass interval. In particular, it can be immediately seen that $\left\|A_{C}^{R} f\right\|_{X_{C}^{R}} \leq \alpha(R)\|f\|_{X_{C}^{R}}$ for all $f \in X_{C}^{R}$. The $A_{C}^{R}$-boundedness of $B_{C}^{R}$ can be derived from a standard argument involving the direct integration of $B_{C}^{R} f$ over the interval $(N, R)$ with respect to the measure $x \mathrm{~d} x$. This is followed by a change in the order of integration, which after employing the mass conservation condition (1.3) provides us with $\left\|B_{C}^{R} f\right\|_{X_{C}^{R}} \leq\left\|A_{C}^{R} f\right\|_{X_{C}^{R}}$. Full details of the derivation can be found in Lemma 6.1.1 of [4]. The $A_{C}^{R}$-boundedness of $C_{R}$ follows by a similar operation, with the weighted sum norm of $X_{D}$ replacing the integral norm of $X_{C}^{R}$. Details of an equivalent operation can be found in Lemma 4.2 of [5].

Recalling that the space $X_{D}$ is finite-dimensional, the linear operators $A_{D}$ and $B_{D}$ are necessarily bounded. Therefore, together with Lemma 6.1, we see that the operator sums $A_{C}^{R}+B_{C}^{R}$ and $A_{D}+B_{D}$ are well defined operators on the entirety of $X_{C}^{R}$ and $X_{D}$ respectively. Likewise, $C_{R}$ is well defined with $D\left(C_{R}\right)=X_{C}^{R}$.

Having considered our continuous and discrete problems separately until this point, we now combine (6.1) and (6.2) within a unified framework, writing them as the following abstract Cauchy problem on the product space $X_{D} \times X_{C}^{R}$ :

$$
\frac{\mathrm{d}}{\mathrm{d} t} \boldsymbol{u}^{R}(t)=\boldsymbol{A}_{R}\left[\boldsymbol{u}^{R}(t)\right], \quad t>0 ; \quad \boldsymbol{u}^{R}(0)=\boldsymbol{u}_{0}^{R} \in D\left(\boldsymbol{A}_{R}\right)=X_{D} \times X_{C}^{R},
$$


where $\boldsymbol{u}^{R}(t), \boldsymbol{A}_{R}$ and $\boldsymbol{u}_{0}^{R}$ are given by

$$
\boldsymbol{u}^{R}(t)=\left(\begin{array}{c}
u_{D}^{R}(t) \\
u_{C}^{R}(t)
\end{array}\right), \quad \boldsymbol{A}_{R}=\left(\begin{array}{cc}
A_{D}+B_{D} & C_{R} \\
0_{D C_{R}} & A_{C}^{R}+B_{C}^{R}
\end{array}\right), \quad \boldsymbol{u}_{0}^{R}=\left(\begin{array}{c}
d_{0} \\
c_{0}^{R}
\end{array}\right)
$$

and where the subscripts on the zero operator $0_{D C_{R}}$ indicate that it maps from $X_{D}$ to $X_{C}^{R}$. It is worth noting that due to the boundedness of each of its components, the operator matrix $\boldsymbol{A}_{R}$ is itself a bounded linear operator on the space $X_{D} \times X_{C}^{R}$. As such, it must generate a uniformly continuous semigroup $\left(\boldsymbol{T}_{R}(t)\right)_{t \geq 0}$ on $X_{D} \times X_{C}^{R}$, ([25], Chap. 1, Thm. 3.7 and Chap. 2, Cor. 1.5), providing us with a unique strong solution to the abstract Cauchy problem (6.3) via $\boldsymbol{u}^{R}(t)=\boldsymbol{T}_{R}(t) \boldsymbol{u}_{0}^{R}$.

\subsection{Uniqueness and differentiability of solutions}

Although the existence of a unique strong solution $\boldsymbol{u}^{R}(t)$ to (6.3) follows immediately from the status of $\boldsymbol{A}_{R}$ as a generator, in the analysis to follow we scrutinise the semigroup $\left(\boldsymbol{T}_{R}(t)\right)_{t>0}$ and its generator, seeking to determine the form taken by this solution, its properties and its relationship to the solutions of Sections 5.2 and 5.4. The following lemma examines the operators from the main diagonal of $\boldsymbol{A}_{R}$, establishing them as generators in their own right.

Lemma 6.2. The operators $A_{C}^{R}+B_{C}^{R}$ and $A_{D}+B_{D}$ generate uniformly continuous semigroups of positive contractions on the spaces $X_{C}^{R}$ and $X_{D}$ respectively.

Proof. As a bounded linear operator on the space $X_{C}^{R}$, the sum $A_{C}^{R}+B_{C}^{R}$ generates a uniformly continuous semigroup $\left(T_{R}(t)\right)_{t>0}$ on $X_{C}^{R}$. Furthermore, the Kato-Voigt perturbation theorem, Corollary 5.17 of [8] as applied in Theorem 3.2 of [5], is readily utilised in the case $\left(A_{C}^{R}+B_{C}^{R}, X_{C}^{R}\right)$ to give us an "extension" of $\left(A_{C}^{R}+B_{C}^{R}, X_{C}^{R}\right)$ as a generator of a substochastic semigroup. Now as $A_{C}^{R}+B_{C}^{R}$ is defined and bounded on all of $X_{C}^{R}$, this extension must be $A_{C}^{R}+B_{C}^{R}$ itself and by Theorem 2.6 of [47], the substochastic semigroup generated must be $\left(T_{R}(t)\right)_{t \geq 0}$. Hence $\left(T_{R}(t)\right)_{t>0}$ is a uniformly continuous semigroup of positive contractions.

By an identical argument, the boundedness of the operators $A_{D}$ and $B_{D}$ means their sum must generate a uniformly continuous semigroup $(T(t))_{t>0}$ on $X_{D}$. We note that in [41], a fully discrete fragmentation model was examined in a weighted $\ell_{1}$ space via the Kato-Voigt perturbation theorem, and (an extension of) the fragmentation operator was found to generate a substochastic semigroup. We can think of infinitely extending our finite discrete fragmentation operators $A_{D}$ and $B_{D}$ by zero to form operators in the $\ell_{1}$ space of [41]. This extension exercise forms an isometry between our discrete space $X_{D}$ and a subspace of the weighted $\ell_{1}$ space, and as such the norms of the semigroups generated must agree between spaces. Since the semigroup in [41] was substochastic, we can therefore surmise that the semigroup $(T(t))_{t \geq 0}$ must also consist of positive contractions.

Having demonstrated that the entries from the main diagonal of $\boldsymbol{A}_{R}$ are generators, and established the nature of their respective semigroups, we are now in a position to consider the unified equation (6.3) and determine the form taken by the semigroup $\left(\boldsymbol{T}_{R}(t)\right)_{t \geq 0}$.

Theorem 6.3. The uniformly continuous semigroup $\left(\boldsymbol{T}_{R}(t)\right)_{t \geq 0}$ generated on $X_{D} \times X_{C}^{R}$ by the operator matrix $\boldsymbol{A}_{R}$ is given by

$$
\boldsymbol{T}_{R}(t)=\left(\begin{array}{cc}
T(t) & Q_{R}(t) \\
0_{D C_{R}} & T_{R}(t)
\end{array}\right), \quad t \geq 0
$$


where $Q_{R}(t): X_{C}^{R} \rightarrow X_{D}$ is defined by $Q_{R}(t) f=\int_{0}^{t} T(t-s) C_{R} T_{R}(s) f \mathrm{~d} s$, for $f \in X_{C}^{R}$. Therefore, the equations (6.2) and (6.1) have unique strong solutions $u_{D}^{R}(t)$ and $u_{C}^{R}(t)$ respectively, which are given by

$$
\begin{aligned}
& u_{D}^{R}(t)=T(t) d_{0}+\int_{0}^{t} T(t-s) C_{R} T_{R}(s) c_{0}^{R} \mathrm{~d} s, \\
& u_{C}^{R}(t)=T_{R}(t) c_{0}^{R} .
\end{aligned}
$$

Proof. Due to the generative nature of $A_{C}^{R}+B_{C}^{R}$ and $A_{D}+B_{D}$ established in Lemma 6.2, and the boundedness of $C_{R}$ established in Lemma 6.1, the result follows immediately from Proposition 3.1 and Corollary 3.2 of $[45]$.

Given the existence of the unique strong solution to the unified abstract Cauchy problem (6.3), we look to determine the relationship between this solution and the weak scalar-valued solutions of Sections 5.2 and 5.4, and in the process hope to ascertain whether the weak solutions may indeed be unique and display extra regularity. However, we first generalise the notion of a solution within the $X_{D} \times X_{C}^{R}$ setting.

\subsubsection{Equivalence of strong and weak solutions in $X_{D} \times X_{C}^{R}$}

The equation (6.3) has a unique strong solution given by $\boldsymbol{u}^{R}(t)=\boldsymbol{T}_{R}(t) \boldsymbol{u}_{0}^{R}$; additionally by Chapter 2, Proposition 6.4 of [25] this is also a unique mild solution, satisfying an equation of the form

$$
\boldsymbol{u}^{R}(t)=\boldsymbol{u}_{0}^{R}+\boldsymbol{A}_{R} \int_{0}^{t} \boldsymbol{u}^{R}(s) \mathrm{d} s=\boldsymbol{u}_{0}^{R}+\int_{0}^{t} \boldsymbol{A}_{R} \boldsymbol{u}^{R}(s) \mathrm{d} s .
$$

We are able to take the operator $\boldsymbol{A}_{R}$ inside the integral as a consequence of its boundedness by applying Proposition 1.1.7 of [2]. Hence our equation (6.7) corresponds with the mild solution form of Definition 1.10 from [19]. The article [19] also provides a notion of an $X_{D} \times X_{C}^{R}$-valued weak solution of equations of type (6.3), which we outline here for our specific example.

Definition 6.4. The function $\boldsymbol{u}^{R}:[0, T) \longrightarrow X_{D} \times X_{C}^{R}$ is a weak solution of (6.3), if for all $\phi$ in $\left(X_{D} \times X_{C}^{R}\right)^{\prime}=$ $X_{D}{ }^{\prime} \times X_{C}^{R^{\prime}}$, we have that $t \rightarrow\left\langle\boldsymbol{u}^{R}(t), \phi\right\rangle$ is locally integrable in $(0, T)$ and

$$
\int_{0}^{T}\left\langle\boldsymbol{u}^{R}(s), \phi\right\rangle \frac{\mathrm{d}}{\mathrm{d} s} \psi(s) \mathrm{d} s=-\left\langle\boldsymbol{u}_{0}^{R}, \phi\right\rangle \psi(0)-\int_{0}^{T}\left\langle\boldsymbol{A}_{R} \boldsymbol{u}^{R}(s), \phi\right\rangle \psi(s) \mathrm{d} s,
$$

for all $\psi \in C_{c}^{\infty}([0, T))$, where $\langle g, \phi\rangle$ denotes the duality pairing of $g$ and $\phi$.

Remark 6.5. If $\mathcal{D} \subseteq X_{D}{ }^{\prime} \times X_{C}^{R^{\prime}}$ is dense in the weak-* topology, then it is sufficient to show that (6.8) holds for all $\phi \in \mathcal{D}$ to establish $\boldsymbol{u}^{R}:[0, T) \rightarrow X_{D} \times X_{C}^{R}$ as a weak solution of (6.3); see Definition 1.16 of [19].

The results so far have provided us with the existence of unique strong and mild solutions to (6.3). We now show that, for our case, any mild solution satisfying (6.7) must necessarily be a weak solution as in Definition 6.4 and vice-versa, providing the existence and uniqueness of a weak solution.

Theorem 6.6. The function $\boldsymbol{u}^{R}:[0, \infty) \longrightarrow X_{D} \times X_{C}^{R}$ provided by the semigroup $\left(\boldsymbol{T}_{R}(t)\right)_{t \geq 0}$ is the unique (integrable) weak solution to equation (6.3), satisfying Definition 6.4 over any time interval $[0, T)$ where $T<\infty$.

Proof. From the analysis above we have the semigroup $\left(\boldsymbol{T}_{R}(t)\right)_{t \geq 0}$ providing a unique mild (strong) solution to equation (6.3) via $\boldsymbol{u}^{R}(t)=\boldsymbol{T}_{R}(t) \boldsymbol{u}_{0}^{R}$. From standard results, it is easily established that for such a $\boldsymbol{u}^{R}$, the right-hand side of $(6.3)$ is integrable over $[0, T)$ for any $T<\infty$. The result Theorem 1.20 of [19] tells us that if the right-hand side of (6.3) is integrable, then given either a mild or weak solution to our evolution equation, the solution can be modified on a set of measure zero to obtain a solution of the other form. Therefore our 
mild solution $\boldsymbol{u}^{R}$ acts as a weak solution as per Definition 6.4. Now suppose that $\boldsymbol{u}^{R}:[0, T) \longrightarrow X_{D} \times X_{C}^{R}$ is an integrable weak solution of (6.3), satisfying equation (6.8). The boundedness of $\boldsymbol{A}_{R}$, together with the integrability of $\boldsymbol{u}^{R}$ ensures that the right-hand side of (6.3) is integrable over $[0, T)$. Therefore, our weak solution must coincide with our unique mild solution for almost all $t \in[0, T)$, hence it must itself be unique and futhermore agree with the strong solution given by the semigroup $\left(\boldsymbol{T}_{R}(t)\right)_{t \geq 0}$.

\subsubsection{Relating scalar-valued weak solutions and weak solutions in $X_{D} \times X_{C}^{R}$}

Having established the existence of a unique solution to the weak formulation (6.8), we look to express the two components of equation (6.8) in a more tangible format, enabling comparison with the scalar-valued weak formulations (5.3) and (5.7) of Section 5. In the first instance we consider the component of (6.8) relating to the continuous fragmentation regime. Let $u_{C}^{R}(x, t)$ be a scalar representation of $\left(u_{C}^{R}(t)\right)(x)=\left(T_{R}(t) c_{0}^{R}\right)(x)$, which forms the continuous component of the solution $\boldsymbol{u}^{R}(t)=\boldsymbol{T}_{R}(t) \boldsymbol{u}_{0}^{R}$, then noting the equivalence of (1.5) and (1.6) as detailed fully in Appendix C of [4], we get that the $X_{C}^{R}$ component of equation (6.8) is equivalent to the following equation:

$$
\begin{aligned}
\int_{0}^{T} & \int_{N}^{R} x u_{C}^{R}(x, t) \frac{\partial \varphi}{\partial t}(x, t) \mathrm{d} x \mathrm{~d} t+\int_{N}^{R} x u_{C}^{R}(x, 0)(x) \varphi(x, 0) \mathrm{d} x \\
& =\int_{0}^{T} \int_{N}^{R} \mathcal{F}^{R}\left(x u_{C}^{R}(x, t)\right)(x, t) \frac{\partial \varphi}{\partial x}(x, t) \mathrm{d} x \mathrm{~d} t+\int_{0}^{T} \int_{N}^{R} S\left(x u_{C}^{R}(x, t)\right)(x, t) \varphi(x, t) \mathrm{d} x \mathrm{~d} t,
\end{aligned}
$$

for all $\varphi$ of the form $\varphi(x, t)=\phi(x) \psi(t)$ where $\phi \in C_{c}^{\infty}((N, R))$ and $\psi \in C_{c}^{\infty}([0, T))$, due to the weak-* density of $C_{c}^{\infty}((N, R))$ in $L_{\infty}((N, R))=X_{C}^{R^{\prime}}$, via Remark 6.5.

Now suppose $u_{D}^{R}:[0, T) \rightarrow X_{D}$ is the discrete component of the solution $\boldsymbol{u}^{R}(t)=\boldsymbol{T}_{R}(t) \boldsymbol{u}_{0}^{R}$, then denoting the individual components of $u_{D}^{R}$ by $u_{D i}^{R}:[0, T) \rightarrow \mathbb{R}$ for $i=1, \ldots, N$, we see that the discrete part of equation (6.8) is equivalent to the following equation:

$$
\begin{aligned}
\sum_{i=1}^{N} \phi_{i} & \int_{0}^{T} u_{D i}^{R}(t) \frac{\mathrm{d}}{\mathrm{d} t} \psi(t) \mathrm{d} t=-\sum_{i=1}^{N} \phi_{i} d_{0 i} \psi(0)+\sum_{i=1}^{N} \phi_{i} \int_{0}^{T} a_{i} u_{D i}^{R}(t) \psi(t) \mathrm{d} t \\
& -\sum_{i=1}^{N} \phi_{i} \int_{0}^{T} \sum_{j=i+1}^{N} a_{j} b_{i, j} u_{D j}^{R}(t) \psi(t) \mathrm{d} t-\sum_{i=1}^{N} \phi_{i} \int_{0}^{T} \int_{N}^{R} a(y) b_{i}(y) u_{C}^{R}(y, t) \psi(t) \mathrm{d} y \mathrm{~d} t,
\end{aligned}
$$

for any $\phi=\left(\phi_{1}, \ldots, \phi_{N}\right) \in \mathbb{R}^{N}=X_{D}{ }^{\prime}$ and $\psi \in C_{c}^{\infty}([0, T))$, where $u_{C}^{R}(\cdot, t)$ is a real-valued representation of the $X_{C}^{R}$-valued $u_{C}^{R}:[0, T) \rightarrow X_{C}^{R}$.

Theorem 6.7. The weak solutions obtained as the limit of the sequence of approximate solutions for the continuous regime in Theorems 5.3 and 5.9, and the discrete regime in Theorems 5.11 and 5.14 are unique, continuously differentiable with respect to $t$ on any interval $[0, T)$ and satisfy equations (1.5) and (1.8) directly.

Proof. It is easily seen that since our scalar-valued continuous weak solution $u_{C}^{R}$ from Section 5 satisfies Definition 5.5, it immediately satisfies the above equation (6.9). From such a scalar-valued function we may then define a function $u_{C}^{R}:[0, T) \longrightarrow X_{C}^{R}$ via $\left(u_{C}^{R}(t)\right)(x):=u_{C}^{R}(x, t)$, for almost all $(x, t) \in(N, R) \times[0, T)$, which satisfies the $X_{C}^{R}$ component of (6.8). Considering the discrete regime, and comparing (6.10) with equation (5.7) from Definition 5.13, it is easily seen that the set of $\left\{u_{D i}^{R}\right\}$ obtained in Section 5, provide us with a solution to the above equation (6.10). As such, taking these $u_{D i}^{R}:[0, T) \rightarrow \mathbb{R}, i=1, \ldots, N$, as the components of the function $u_{D}^{R}:[0, T) \rightarrow X_{D}$, we obtain the $X_{D}$ component of a solution to equation (6.8). Therefore, taken together the scalar-valued weak solutions from Section 5 provide us with an $X_{D} \times X_{C}^{R}$-valued weak solution $\boldsymbol{u}^{R}$ to (6.8). Furthermore, as the scalar-valued solutions were obtained as weak limits of bounded sequences in $L_{1}((N, R) \times[0, T), x \mathrm{~d} x \mathrm{~d} t)$ and $L_{1}([0, T))$, they produce a $\boldsymbol{u}^{R}$ which is integrable. By Theorem 6.6, any such 
$\boldsymbol{u}^{R}$ must be unique (up to sets of measure zero) and coincide with the strong solution provided by the semigroup $\left(\boldsymbol{T}_{R}(t)\right)_{t \geq 0}$. As such, our scalar-valued solutions $u_{C}^{R}(x, t)$ and $u_{D i}^{R}(t)(i=1, \ldots, N)$ must also be unique (up to sets of measure zero). Furthermore, as a representation of a strongly differentiable $X_{C}^{R}$-valued function, by Theorem 2.40 of [8], the scalar-valued $u_{C}^{R}(x, t)$ is continuously differentiable with respect to $t$, except perhaps on a set of zero measure, and by a similar argument as applied at the end of Theorem 8.3 from [8] or directly as in Theorem 3.2.7 of [4], $u_{C}^{R}(x, t)$ can be seen to directly satisfy equation (1.5) almost everywhere. Likewise the functions $u_{D i}^{R}:[0, T) \rightarrow \mathbb{R}$ must be differentiable in the traditional sense and satisfy (1.8).

The results of this section are stronger than is typical within the literature applying finite volume schemes to fragmentation problems. Generally we are only provided with (weak) convergence to a weak solution, with the potential for multiple solutions arising from different (weakly) convergent subsequences. However, by relating our limit solutions (in a one-to-one manner) with the strong solution to (6.3), we have demonstrated the limit solution must necessarily be unique, continuously differentiable with respect to $t$ and a solution, in the classical sense, of the truncated fragmentation problem. We now consider the truncation error introduced by restricting the continuous mass domain to $(N, R)$. Letting $R \rightarrow \infty$, we will show that the solution $\boldsymbol{u}^{R}$ of the system (6.3) converges to the solution of an equivalent nontruncated abstract Cauchy problem ([5], Eq. (5.1)), whilst also establishing a bound on the error between the two solutions.

\subsection{Truncation error and convergence as $R \rightarrow \infty$}

In the previous section we utilised the operator matrix $\boldsymbol{A}_{R}$ to cast the system (1.5) and (1.8) as the abstract Cauchy problem (6.3) in the product space $X_{D} \times X_{C}^{R}$. In the article [5], a similar approach was used in order to formulate the full system (1.1) and (1.2) in the product space $X_{D} \times X_{C}$, where $X_{C}=L_{1}((N, \infty), x \mathrm{~d} x)$. We now examine the link between these two problems, aiming to show that as we let $R \rightarrow \infty$, the solution $\boldsymbol{u}^{R}$ to (6.3) converges to the solution obtained in [5]. To begin we briefly summarise the necessary concepts, notations and results from [5].

In its handling of the continuous equation (1.1), the article [5] adopts an approach similar to that of Chapter 8 from [8], introducing the following expressions based on the terms of equation (1.1):

$$
(\mathcal{A} f)(x)=-a(x) f(x) \text { and }(\mathcal{B} f)(x)=\int_{x}^{\infty} a(y) b(x \mid y) f(y) \mathrm{d} y \text { for } x>N .
$$

From which we obtain operators $A_{C}$ and $B_{C}$ as follows:

$$
\begin{array}{ll}
\left(A_{C} f\right)(x)=(\mathcal{A} f)(x), & D\left(A_{C}\right)=\left\{f \in X_{C}: A_{C} f \in X_{C}\right\}, \\
\left(B_{C} f\right)(x)=(\mathcal{B} f)(x), & D\left(B_{C}\right)=\left\{f \in X_{C}: B_{C} f \in X_{C}\right\} .
\end{array}
$$

Employing an argument along the lines of Lemma 6.1 , it is easily seen that $D\left(A_{C}\right) \subseteq D\left(B_{C}\right)$ as $\left\|B_{C} f\right\|_{X_{C}} \leq$ $\left\|A_{C} f\right\|_{X_{C}}$ for $f \in D\left(A_{C}\right)$, allowing us to consider the sum $A_{C}+B_{C}$ on the domain $D\left(A_{C}\right)$. For full details see Lemma 3.1 of [5].

We may then consider re-framing (1.1) as an abstract Cauchy problem in $X_{C}$, as was carried out for (1.5) in the space $X_{C}^{R}$ with (6.1). However, due to the unbounded nature of the continuous mass domain in $X_{C}$, the operators $A_{C}$ and $B_{C}$ are no longer bounded as was the case with $A_{C}^{R}$ and $B_{C}^{R}$, and therefore it is not immediate that the sum $A_{C}+B_{C}$ forms a generator. However, an application of the Kato-Voigt perturbation theorem ([8], Cor. 5.17), provides the existence of an extension $(K, D(K))$ of $\left(A_{C}+B_{C}, D\left(A_{C}\right)\right)$, that generates a substochastic semigroup $\left(G_{K}(t)\right)_{t>0}$ on $X_{C}$, see Theorem 3.2 of [5] for the details. In Theorems 3.4 and 3.5 of [5], by following the approach of Theorem 8.5 from [8], it was determined that the generator $(K, D(K))$ is in fact the closure of $\left(A_{C}+B_{C}, D\left(A_{C}\right)\right)$ in $X_{C}$, establishing the honesty of the semigroup $\left(G_{K}(t)\right)_{t \geq 0}$ in the process. The equation (1.1) was then expressed as the following abstract Cauchy problem in $X_{C}$ :

$$
\frac{\mathrm{d}}{\mathrm{d} t} u_{C}(t)=K\left[u_{C}(t)\right], \quad t>0 ; \quad u_{C}(0)=c_{0} \in D(K) .
$$


In [5], the analysis of the discrete system (1.2) was conducted within the same space $X_{D}$ as used for (1.8). Furthermore, the same discrete fragmentation operators $A_{D}$ and $B_{D}$, as appear in (6.2), were used in [5]. However, we must introduce the operator $C: D(C) \subseteq X_{C} \rightarrow X_{D}$, as the counterpart to $C_{R}$, and which is given elementwise by

$$
(C f)_{i}=\int_{N}^{\infty} a(y) b_{i}(y) f(y) \mathrm{d} y, \quad D(C)=\left\{f \in X_{C}: C f \in X_{D}\right\} .
$$

The system (1.2) then becomes

$$
\frac{\mathrm{d}}{\mathrm{d} t} u_{D}(t)=\left(A_{D}+B_{D}\right)\left[u_{D}(t)\right]+C\left[u_{C}(t)\right], \quad t>0 ; \quad u_{D}(0)=d_{0},
$$

As with (6.1) and (6.2), the equations (6.11) and (6.12) are unified as a single system, this time as the following abstract Cauchy problem in the product space $X_{D} \times X_{C}$ :

$$
\frac{\mathrm{d}}{\mathrm{d} t} \boldsymbol{u}(t)=\boldsymbol{A}[\boldsymbol{u}(t)], \quad t>0 ; \quad \boldsymbol{u}(0)=\boldsymbol{u}_{0} \in D(\boldsymbol{A})=X_{D} \times D(K),
$$

where $\boldsymbol{u}(t), \boldsymbol{A}$ and $\boldsymbol{u}_{0}$ are given by

$$
\boldsymbol{u}(t)=\left(\begin{array}{l}
u_{D}(t) \\
u_{C}(t)
\end{array}\right), \quad \boldsymbol{A}=\left(\begin{array}{cc}
A_{D}+B_{D} & C \\
0_{D C} & K
\end{array}\right), \quad \boldsymbol{u}_{0}=\left(\begin{array}{c}
d_{0} \\
c_{0}
\end{array}\right)
$$

By employing a similar argument as adopted in Theorem 6.3, in Theorem 5.2 of [5] the operator $\boldsymbol{A}$ is found to be a generator, providing a unique strong solution to the equation (6.13) through the semigroup

$$
\boldsymbol{T}(t)=\left(\begin{array}{cc}
T(t) & Q(t) \\
0_{D C} & G_{K}(t)
\end{array}\right), \quad t \geq 0
$$

where $Q(t): D(K) \subseteq X_{C} \rightarrow X_{D}$ is defined by $Q(t) f=\int_{0}^{t} T(t-s) C G_{K}(s) f \mathrm{~d} s$, for $f \in D(K)$. Therefore, the equations (6.12) and (6.11) have unique strong solutions $u_{D}(t)$ and $u_{C}(t)$ respectively, which are given by

$$
\begin{aligned}
& u_{D}(t)=T(t) d_{0}+\int_{0}^{t} T(t-s) C G_{K}(s) c_{0} \mathrm{~d} s, \\
& u_{C}(t)=G_{K}(t) c_{0} .
\end{aligned}
$$

In order to analyse the truncation error between the solution $\boldsymbol{u}^{R}$ to (6.3) and the solution $\boldsymbol{u}$ of (6.13), it is necessary to lift (6.3) from $X_{D} \times X_{C}^{R}$ into $X_{D} \times X_{C}$. As the truncation error originates from the restriction of the continuous mass domain $(N, \infty)$ to $(N, R)$, the error between the solutions $\boldsymbol{u}^{R}$ and $\boldsymbol{u}$ is heavily dependent upon their continuous components, namely $u_{C}^{R}$ from (6.6) and $u_{C}$ from (6.16). Therefore we begin by transporting $u_{C}^{R}$ to $X_{C}$ and establishing the convergence of $u_{C}^{R}$ to $u_{C}$ as $R \rightarrow \infty$. We do not cover the full details of this convergence argument here owing to its length and the similarity with the approach set out in Section 8.3.2 of [8]. However, we now highlight some key points from that argument that will allow us to derive a bound on the truncation error. Full details of the convergence result in the specific case considered here may be found in Chapter 6 of [4]. In order to examine the convergence of the truncated solution $u_{C}^{R}$ to the full solution $u_{C}$, we introduce the following family of projection operators $\left\{P_{R}\right\}$, which act on $X_{C}$ and are defined as follows. For $f \in X_{C}$ we have

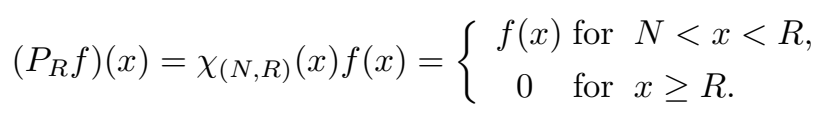


Each projection operator maps the space $X_{C}$ onto the subspace of $X_{C}$ consisting of those elements which are zero over the interval $[R, \infty)$. This subspace is isometrically isomorphic to the space $X_{C}^{R}$, and for our purposes we may consider the spaces to be the same. We also introduce the notation $K_{R}$ to denote the sum $A_{C}^{R}+B_{C}^{R}$ as the generator of $\left(T_{R}(t)\right)_{t \geq 0}$. The following lemma outlines a number of properties of the semigroup $\left(T_{R}(t)\right)_{t \geq 0}$ and generator $K_{R}$, which we shall utilise in the upcoming analysis.

Lemma 6.8. The family of semigroups $\left(T_{R}(t)\right)_{t \geq 0}$ and their generators $K_{R}$ satisfy the following properties:

(i) For any $\tilde{R} \geq R>N$ and $t \geq 0, P_{R} T_{\tilde{R}}(t) P_{R}=T_{R}(t)$. In particular we have $P_{R} T_{R}(t) P_{R}=T_{R}(t)$ for all $R>N$.

(ii) $K P_{R}=P_{R} K P_{R}=K_{R}$ for all $R>N$.

Proof. Both properties can be readily established by considering the characteristics of the projection operators, however details can be found in Lemma 8.6 of [8] and Lemma 6.1.5 of [4].

Although the semigroup $\left(T_{R}(t)\right)_{t \geq 0}$ acts on $X_{C}^{R}$, it can be extended to act on $X_{C}$ in the following manner:

$$
\bar{T}_{R}(t)=P_{R} T_{R}(t) P_{R}
$$

The resulting family of operators $\left(\bar{T}_{R}(t)\right)_{t>0}$ is uniformly continuous, however, it does not form a semigroup on $X_{C}$, since setting $t=0$ does not produce the identity operator on $X_{C}$. Additionally, since the operator $K_{R}$ is itself a bounded operator on $X_{C}$, it must generate a uniformly continuous semigroup on $X_{C}$, which we denote by $\left(S_{R}(t)\right)_{t \geq 0}$. In the final lemma of this section we determine a number of key properties of the families $\left(T_{R}(t)\right)_{t \geq 0},\left(\bar{T}_{R}(t)\right)_{t \geq 0}$ and $\left(S_{R}(t)\right)_{t \geq 0}$, including their behaviour as $R \rightarrow \infty$.

Lemma 6.9. The operator families $\left(T_{R}(t)\right)_{t \geq 0},\left(\bar{T}_{R}(t)\right)_{t \geq 0}$ and $\left(S_{R}(t)\right)_{t \geq 0}$ satisfy the following properties:

(i) The family of operators $\left(\bar{T}_{R}(t)\right)_{t \geq 0}$ is increasing with $R$. That is, for $f \in X_{C+}$ and each fixed $t \geq 0$ we have

$$
\bar{T}_{\tilde{R}}(t) f \geq \bar{T}_{R}(t) f, \text { when } \tilde{R} \geq R .
$$

(ii) There exists a strongly continuous semigroup $(G(t))_{t \geq 0}$ of positive contractions on $X_{C}$, such that for $f \in X_{C}$ and $t \geq 0$,

$$
G(t) f=\lim _{R \rightarrow \infty} \bar{T}_{R}(t) f=\lim _{R \rightarrow \infty} S_{R}(t) f .
$$

Additionally, the convergence in both limits is uniform with respect to $t$, on bounded intervals. Further, when $f \in X_{C}^{\tilde{R}}$, we have

$$
G(t) f=P_{R} T_{R}(t) P_{R} f,
$$

for any $R \geq \tilde{R}$.

(iii) Under the assumptions so far imposed, the semigroups $(G(t))_{t \geq 0}$ and $\left(G_{K}(t)\right)_{t \geq 0}$ agree on $X_{C}$, that is

$$
G(t) f=G_{K}(t) f, \quad t \geq 0, \quad f \in X_{C} .
$$

Proof. For properties (i) and (ii) see Proposition 8.8 of [8] or alternatively Lemma 6.1.6 of [4] and Theorem 6.1 .8 of [4] respectively. Whilst for property (iii) the reader should consult Proposition 8.10 of [8] or Theorem 6.1 .10 of [4].

Having set out these results we are now in a position to derive a bound on the error introduced by truncating our mass domain, that is the discrepancy between the solution $\boldsymbol{u}^{R}$ to (6.3) and the full solution $\boldsymbol{u}$ which satisfies (6.13). 
Theorem 6.10. The truncation error between $\boldsymbol{u}^{R}(t)$ and $\boldsymbol{u}(t)$, as measured by the norm on $X_{D} \times X_{C}$, remains constant for all $t \geq 0$, hence is equal to the truncation error induced by truncating the initial mass distribution of the continuous regime, that is

$$
\left\|\boldsymbol{u}(t)-\boldsymbol{u}^{R}(t)\right\|_{X_{D} \times X_{C}}=\left\|\boldsymbol{u}_{0}-\boldsymbol{u}_{0}^{R}\right\|_{X_{D} \times X_{C}}=\left\|c_{0}-c_{0}^{R}\right\|_{X_{C}}, \quad \text { for all } t \geq 0 .
$$

Therefore, for all $t \geq 0$, the truncated solution $\boldsymbol{u}^{R}(t)$ converges to $\boldsymbol{u}(t)$ in $X_{D} \times X_{C}$ as $R \rightarrow \infty$.

Proof. From property (i) of Lemma 6.8 and properties (ii) and (iii) of Lemma 6.9, it is easily seen that since $c_{0}^{R} \in X_{C}^{R}$, we have

$$
T_{R}(t) c_{0}^{R}=P_{R} T_{R}(t) P_{R} c_{0}^{R}=G(t) c_{0}^{R}=G_{K}(t) c_{0}^{R} .
$$

Due to idempotence of the projection operators, the first part of (6.17) also gives us $T_{R}(s) c_{0}^{R}=P_{R} T_{R}(s) c_{0}^{R}$. Whilst for $f \in X_{C}^{R}$, it readily follows from the definition of the operators $C_{R}$ and $C$, in addition to the projections $P_{R}$, that $C_{R} f=C P_{R} f$. Therefore, considering the application of the operator $C_{R}$ to $T_{R}(t) c_{0}^{R}$, we get

$$
C_{R} T_{R}(s) c_{0}^{R}=C P_{R} T_{R}(s) c_{0}^{R}=C T_{R}(s) c_{0}^{R}=C G_{K}(t) c_{0}^{R},
$$

where the last equality follows from another application of (6.17). Utilising the relations (6.17) and (6.18) allows us to re-write the components of $\boldsymbol{u}^{R}$, given by (6.5) and (6.6), as follows:

$$
\begin{aligned}
& u_{D}^{R}(t)=T(t) d_{0}+\int_{0}^{t} T(t-s) C G_{K}(s) c_{0}^{R} \mathrm{~d} s, \\
& u_{C}^{R}(t)=G_{K}(t) c_{0}^{R} .
\end{aligned}
$$

Taking the difference between these components and those of $\boldsymbol{u}$ as given by (6.15) and (6.16), provides the error $\boldsymbol{u}-\boldsymbol{u}^{R}=: \boldsymbol{u}_{E}^{R}$, expressed in component form as follows:

$$
\begin{aligned}
u_{D}(t)-u_{D}^{R}(t) & =T(t) d_{0}+\int_{0}^{t} T(t-s) C G_{K}(s) c_{0} \mathrm{~d} s-T(t) d_{0}-\int_{0}^{t} T(t-s) C G_{K}(s) c_{0}^{R} \mathrm{~d} s \\
& =T(t)\left(d_{0}-d_{0}\right)+\int_{0}^{t} T(t-s) C G_{K}(s)\left(c_{0}-c_{0}^{R}\right) \mathrm{d} s, \\
u_{C}(t)-u_{C}^{R}(t) & =G_{K}(t) c_{0}-G_{K}(t) c_{0}^{R}=G_{K}(t)\left(c_{0}-c_{0}^{R}\right) .
\end{aligned}
$$

Comparing (6.19) and (6.20) to (6.15) and (6.16), we see that $\boldsymbol{u}_{E}^{R}$ provides a solution to the ACP (6.13) where the initial condition $\boldsymbol{u}_{0}$ is given by

$$
\boldsymbol{u}_{0}=\left(\begin{array}{c}
d_{0}-d_{0} \\
c_{0}-c_{0}^{R}
\end{array}\right)=\left(\begin{array}{c}
0_{D} \\
c_{0}-c_{0}^{R}
\end{array}\right)
$$

Furthermore, as $c_{0}(x) \geq c_{0}^{R}(x)$ for all $x>N$, this initial condition is clearly nonnegative. In Lemmas 6.1 and 6.2 of [5], solutions of the problem (6.13) with nonnegative initial data were seen to remain nonnegative and to conserve mass for $t \geq 0$. As the norm $\left\|\boldsymbol{u}_{E}^{R}(t)\right\|_{X_{D} \times X_{C}}$ corresponds to the total mass accounted for by the solution $\boldsymbol{u}_{E}^{R}$ at time $t$, this mass conservation gives us

$$
\left\|\boldsymbol{u}(t)-\boldsymbol{u}^{R}(t)\right\|_{X_{D} \times X_{C}}=\left\|\boldsymbol{u}_{E}^{R}(t)\right\|_{X_{D} \times X_{C}}=\left\|\boldsymbol{u}_{E}^{R}(0)\right\|_{X_{D} \times X_{C}}=\left\|\left(\begin{array}{c}
0_{D} \\
c_{0}-c_{0}^{R}
\end{array}\right)\right\|_{X_{D} \times X_{C}}=\left\|0_{D}\right\|_{X_{D}}+\left\|c_{0}-c_{0}^{R}\right\|_{X_{C}},
$$

for all $t \geq 0$. As such, the truncation error between $\boldsymbol{u}^{R}(t)$ and $\boldsymbol{u}(t)$ remains constant for all $t$, being equal to the error introduced within the initial mass distributions, with this being given entirely by the error induced by truncating the initial mass distribution within the continuous regime. The convergence of $\boldsymbol{u}^{R}$ to $\boldsymbol{u}$ follows as an immediate consequence of the above relation. 
This completes our analysis of the mixed discrete-continuous fragmentation equation. In this section, we established that the weak solutions obtained in Section 5, as the limit of our numerical approximations, are in fact unique, differentiable solutions of (1.5) and (1.8). Furthermore, we were able to ascertain the error introduced within this solution upon truncation of the continuous mass domain. Finally, as an immediate consequence of the form of this error, we have seen that by letting $R \rightarrow \infty$, we recover the unique solution of the untruncated system (1.1) and (1.2). In the following section we conduct an empirical investigation, with the objective of corroborating the theoretical results of Sections 5 and 6 .

\section{NUMERICAL EXPERIMENTS}

We conclude our study with an empirical investigation of the results established in the previous sections. In particular, we aim to confirm the convergence of our numerical solutions as $h \rightarrow 0$, assess the order of this convergence, and also corroborate the theoretically established truncation error. As a test case for this investigation, we take the power law model as set out in Section 7 of [5], where the continuous equation was defined by

$$
a(x)=x^{\alpha}, \quad \alpha \in \mathbb{R}, \quad \text { and } \quad b(x \mid y)=(\nu+2) \frac{x^{\nu}}{y^{\nu+1}}, \quad-2<\nu \leq 0 .
$$

The discrete equation was specified by the following values for $a_{i}$ and $b_{i, j}$

$$
a_{i}=\left\{\begin{aligned}
0 & \text { for } i=1, \\
i^{\alpha} & \text { for } i=2, \ldots, N, \quad b_{i, j}=\frac{2}{j-1}, \quad i=1, \ldots, N-1, j=i, \ldots, N,
\end{aligned}\right.
$$

and the continuous to discrete distribution functions $b_{i}(y)$ were given by

$$
b_{i}(y)=\frac{i^{\nu+2}-(i-1)^{\nu+2}}{i y^{\nu+1}}, \quad y>N, i=1, \ldots, N .
$$

It can be easily verified that conditions (1.3) and (1.4) are satisfied by these choices.

\subsection{Convergence of numerical solutions as $h \rightarrow 0$}

We begin by testing the convergence of the numerical solutions, established in Section 5. Taking the test model outlined above, we set the parameters $N$ and $R$ at 5 and 15 respectively, and assume a uniform initial mass distribution, with

$$
c_{0}(x)= \begin{cases}1 & \text { for } 5<x<15 \\ 0 & \text { for } x \geq 15\end{cases}
$$

and $d_{0}$ being the $N$-vector consisting entirely of $1^{\prime}$ s. The parameters $\alpha$ and $\nu$ were varied, taking all possible combinations of $\alpha \in\{0.5,0.1,-0.5,-1,-2\}$ and $\nu \in\{0,-0.5,-1,-1.5\}$, with the final time $T$ selected in each case to allow the system to reach a near equilibrium state. The approximate solutions generated by the numerical scheme were compared to the exact solutions derived in Section 7.1 of [5], with the discrepancy being measured by taking the relative error with respect to the norm on $L_{1}\left([0, T), X_{D}\right) \times L_{1}\left([0, T), X_{C}^{R}\right)$. That is, supposing $\boldsymbol{u}_{h}=\left(\underline{u}_{D}^{h}(t), u_{C}^{h}(x, t)\right)$ is our approximation of a solution $\boldsymbol{u}^{R}=\left(\underline{u}_{D}^{R}(t), u_{C}^{R}(x, t)\right)$, then we measure the error via

$$
\operatorname{Error}\left(\boldsymbol{u}_{h} \mid \boldsymbol{u}^{R}\right)=\frac{\left\|\boldsymbol{u}_{h}-\boldsymbol{u}^{R}\right\|_{R}}{\left\|\boldsymbol{u}^{R}\right\|_{R}}
$$

where the norm $\|\cdot\|_{R}$, is given by

$$
\left\|\boldsymbol{u}^{R}\right\|_{R}=\int_{0}^{T} \sum_{i=1}^{N} i\left|u_{D i}^{R}(t)\right| \mathrm{d} t+\int_{0}^{T} \int_{N}^{R}\left|u_{C}^{R}(x, t)\right| x \mathrm{~d} x \mathrm{~d} t .
$$



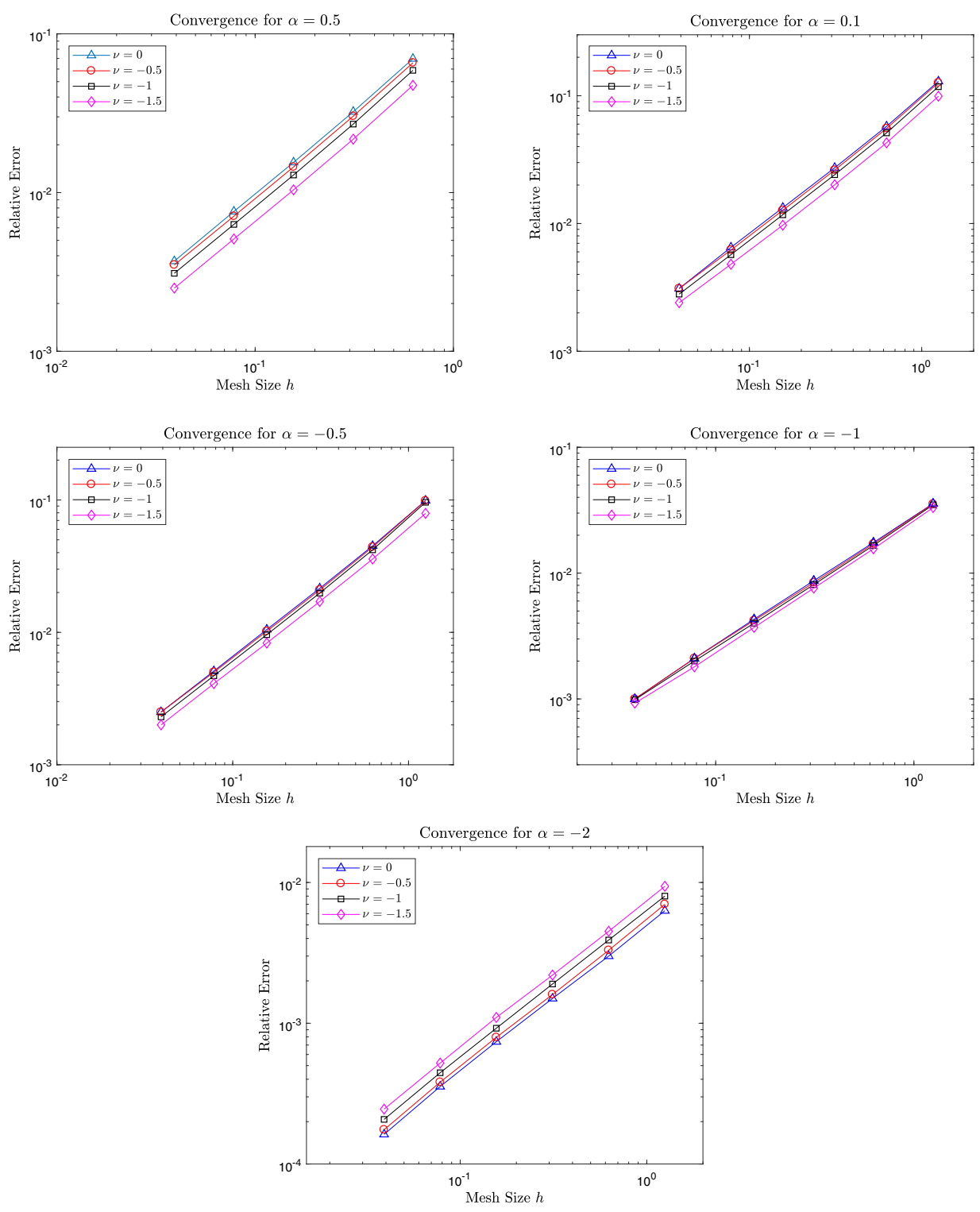

FiguRe 1. Numerical convergence for $\alpha=0.5,0.1,-0.5,-1$ and -2 .

For each model configuration, we computed approximate solutions over a sequence of uniform meshes, refining at each step by halving the mesh parameter $h$. The charts in Figure 1 plot the observed relative error against the mesh parameter $h$, for all possible parameter configurations. From even the briefest examination of the charts it is clear that as the mesh is refined, the relative error of the approximations is reduced. Whilst if we were to examine the gradients of the lines appearing in Figure 1, then they appear generally to be getting closer to 1 , as the mesh is refined. With the gradients between the most refined mesh pairings having a mean value of 1.0301, across all configurations. This would suggest that our numerical scheme has order $\gamma \approx 1$, with the error in the approximations being $\mathcal{O}(h)$. The full numerical details of the errors and the associated convergence rates underlying Figure 1 may be found in Appendix A of [4]. 


\subsection{Convergence of truncated solutions as $R \rightarrow \infty$}

We now turn our attention to the convergence of the truncated solutions as $R \rightarrow \infty$, and in particular the truncation error established in Theorem 6.10. Once again our experimentation is based upon the test model (7.1)-(7.3), with the initial mass distribution unspecified for the moment. Letting $\boldsymbol{u}^{R}=\left(\underline{u}_{D}^{R}(t), u_{C}^{R}(x, t)\right)$ denote the solution based on the interval truncated at $R$ and suppose $\boldsymbol{u}=\left(\underline{u}_{D}(t), u_{C}(x, t)\right)$ is the full solution over the semi-infinite domain, we are interested in measuring the error between $\boldsymbol{u}^{R}$ and $\boldsymbol{u}$. However, the very reason for introducing the truncation was the difficulty in computationally representing the semi-infinite domain of Equation (1.1), a problem now re-encountered in trying to represent the full solution $\boldsymbol{u}$ in our calculations. To circumvent this, we use a truncated solution $\boldsymbol{u}^{R_{\infty}}=\left(\underline{u}_{D}^{R_{\infty}}(t), u_{C}^{R_{\infty}}(x, t)\right)$, based on an interval up to some finite but large $R_{\infty}$, as a stand-in for the full solution $\boldsymbol{u}$. We then consider the relative error between $\boldsymbol{u}^{R}$ and $\boldsymbol{u}^{R_{\infty}}$, as measured by

$$
\operatorname{Error}\left(\boldsymbol{u}^{R} \mid \boldsymbol{u}^{R_{\infty}}\right)=\frac{\left\|\boldsymbol{u}^{R}-\boldsymbol{u}^{R_{\infty}}\right\|_{R_{\infty}}}{\left\|\boldsymbol{u}_{\infty}^{R_{\infty}}\right\|_{R_{\infty}}}
$$

where the norm $\|\cdot\|_{R_{\infty}}$, is the $L_{1}\left([0, T), X_{D}\right) \times L_{1}\left([0, T), X_{C}^{R_{\infty}}\right)$ norm, as specified by (7.4) with $R=R_{\infty}$. From Theorem 6.10 we expect this error to be given by

$$
\operatorname{Error}\left(\boldsymbol{u}^{R} \mid \boldsymbol{u}^{R_{\infty}}\right)=\frac{\left\|c_{0}^{R_{\infty}}-c_{0}^{R}\right\|_{X_{C}^{R \infty}}}{M_{0}} .
$$

where $M_{0}$ signifies the total mass within the initial distribution for $\boldsymbol{u}^{R_{\infty}}$.

As with the previous example, we begin by considering a uniform initial mass distribution, as such

$$
d_{0 i}=b, \text { for } i=1, \ldots, N \text { and } c_{0}(x)=b, \text { for } N<x<R_{\infty} .
$$

The upper limit $R_{\infty}$ is set at 100, and we consider both $N=5$ and $N=25$. In each case the value of $b$ was selected to give an initial total mass of 100 , and hence $b=1.999 \times 10^{-2}$ for $N=5$ and $b=1.995 \times 10^{-2}$ in the case of $N=25$. We considered all combinations for the parameter values $\alpha \in\{0.5,0,-1\}$ and $\nu \in\{0,-1\}$, with the final time $T$ selected in each case so as to allow the system to reach a near equilibrium state. The truncation parameter $R$ was then varied throughout the admissible range, from $N$ up to $R_{\infty}$, taking on the values $R \in\{15,30,40,50,60,70,80,90,95,97,98,99\}$ when $N=5$, with $R=15$ being excluded for the cases with $N=25$.

Carrying out this experimentation we observed that within the set of considered values, the parameters $\alpha$ and $\nu$ had no impact on the empirical values of $\operatorname{Error}\left(\boldsymbol{u}^{R} \mid \boldsymbol{u}^{R_{\infty}}\right)$ as given by (7.5). This is to be expected given (7.6), since the parameters $\alpha$ and $\nu$ do not feature in the initial mass distribution $c_{0}$ and hence do not influence the initial truncation error, and neither do they influence the total initial mass $M_{0}$. Likewise, the relative error (7.5) exhibited no dependence on the regime boundary parameter $N$. The truncation error appearing in the numerator of (7.6) is entirely dictated by the initial mass profile beyond the truncation point $R$, and whilst the total initial mass appearing in the denominator does depend on $N$, we have held this mass constant between the cases. The charts in Figure 2 plot the empirical error $\operatorname{Error}\left(\boldsymbol{u}^{R} \mid \boldsymbol{u}^{R}\right)$, as given by (7.5), on the $y$-axis against the theoretical value $\left\|c_{0}^{R_{\infty}}-c_{0}^{R}\right\|_{X_{C}^{R} \infty} / M_{0}$ along the $x$-axis, with the leftmost chart corresponding to the case $N=5$, and the right chart detailing the results for $N=25$. In both cases, since the parameters $\alpha$ and $\nu$ did not impact our results, we detail only the case of $\alpha=0.5$ and $\nu=0$, the other cases being identical to those shown.

The markers on the charts correspond to the selected values for $R$, with $R$ increasing as we go from the top right to the bottom left of each chart. Noting that as the value of $R$ is increased, the empirically measured $\operatorname{Error}\left(\boldsymbol{u}^{R} \mid \boldsymbol{u}^{R_{\infty}}\right)$ and the theoretical value $\left\|c_{0}^{R_{\infty}}-c_{0}^{R}\right\|_{X_{C}^{R_{\infty}} / M_{0}}$ remain inline with each other, as would be expected given (7.6). The full numerical details of the errors for all experimental configurations may be found in Appendix A of [4]. 

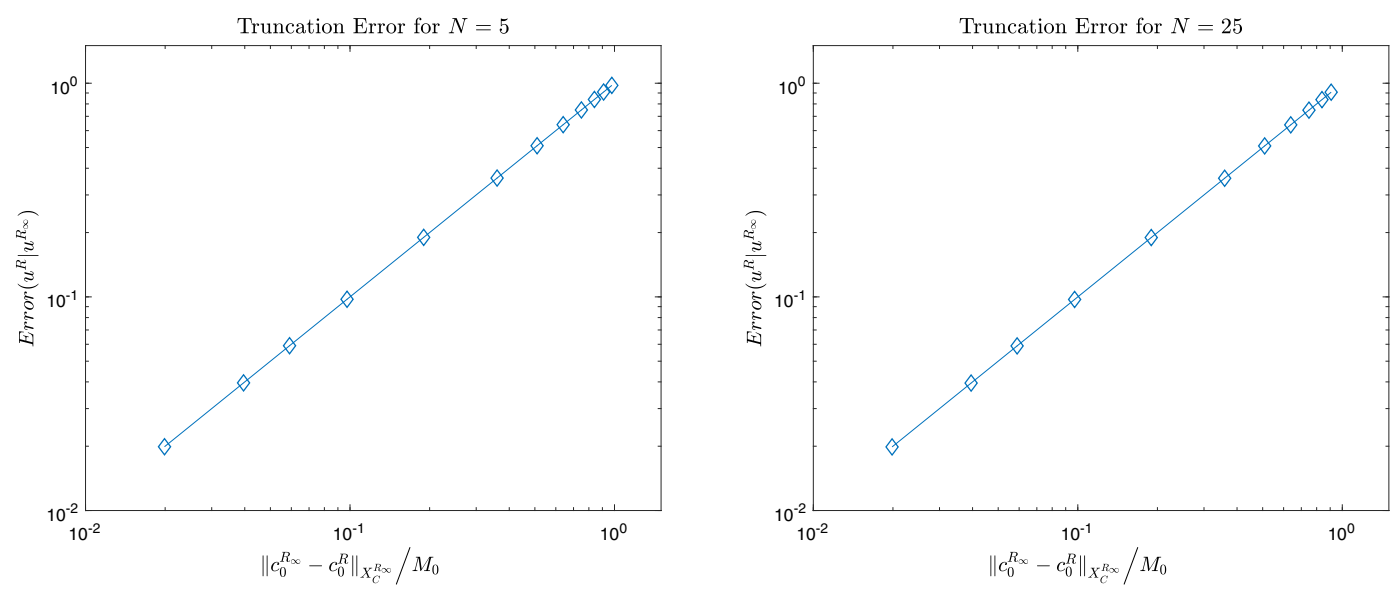

FiguRE 2. Truncation convergence as $R \rightarrow R_{\infty}$ starting from a uniform initial mass distribution, for $N=5$ (left) and $N=25$ (right).

In the previous experiments we imposed a uniform initial mass distribution which was constant up to the truncation point. However, if we consider the full problem (Eqs. (1.1) and (1.2)) with its unbounded domain, then the uniform distribution would violate the requirement for $c_{0}$ to be $L_{1}((N, \infty), x \mathrm{~d} x)$, unless at some point it was indeed truncated. That being the case, we could simply take $R$ to be greater than or equal to the truncation point and obtain the full solution. Therefore, for these next experiments we shall employ an initial mass distribution featuring a negative exponential profile. Such a profile not only allows for infinite support whilst having finite mass, but also fits with the exact solutions to the system (7.1)-(7.3), obtained in [5], which were seen to be driven by the factor $\mathrm{e}^{-x^{\alpha} t}$. Hence, we shall assume an initial state of the form

$$
d_{0 i}=b \mathrm{e}^{c i}, \text { for } i=1, \ldots, N \text { and } c_{0}(x)=b \mathrm{e}^{c x}, \text { for } x>N,
$$

where $b>0$ and $c<0$ are constants to be selected subject to the following criteria. When provided with an $N, R_{\infty}$ and a proportion $p \approx 1$, the parameter $c$ is selected such that the total mass of the initial distribution truncated at $R_{\infty}$ accounts for a proportion $p$ of the total mass of the untruncated distribution with infinite support. The value of $b$ is then selected so that the $R_{\infty}$ truncated distribution has an associated total mass specified by the user.

Once again we conducted a range of experiments considering the same parameter values of $\alpha \in\{-1,0,0.5\}$, $\nu \in\{-1,0\}, N \in\{5,25\}$ and $R_{\infty}=100$. Given these choices of $N$ and $R_{\infty}$, we selected the parameter $c$ such that the initial mass distribution truncated at $R_{\infty}=100$ accounted for a proportion 0.99 of the mass of the untruncated case. The value of $b$ was then chosen to give a total truncated mass (truncated at $R_{\infty}=100$ ) of 100. For $N=5$, this resulted in the choices $b=4.4088 \times 10^{-1}$ and $c=-6.6320 \times 10^{-2}$, whilst $N=25$ gave us $b=4.3985 \times 10^{-1}$ and $c=-6.6320 \times 10^{-2}$. As previously, the truncation parameter $R$ was varied, taking values within the set $\{15,30,40,50,60,70,80,90,95,97,98,99\}$ for $N=5$, with $R=15$ again being excluded when we considered cases with $N=25$.

As was observed in the previous experiments, the parameters $\alpha$ and $\nu$ did not have any impact on the error (7.5), when varied within the considered values, likewise for the truncation parameter $N$. The charts in Figure 3 replicate those of Figure 2, for the case of a negative exponential initial mass distribution. The left-hand chart depicts the results for $N=5$, whilst the right chart details $N=25$. As was the case before, we only consider the configuration of $\alpha=0.5$ and $\nu=0$, owing to the insensitivity of $\operatorname{Error}\left(\boldsymbol{u}^{R} \mid \boldsymbol{u}^{R_{\infty}}\right)$ to these parameters. Examining both charts, we see a similar pattern as before, with equality between the observed empirical error (7.5) and 

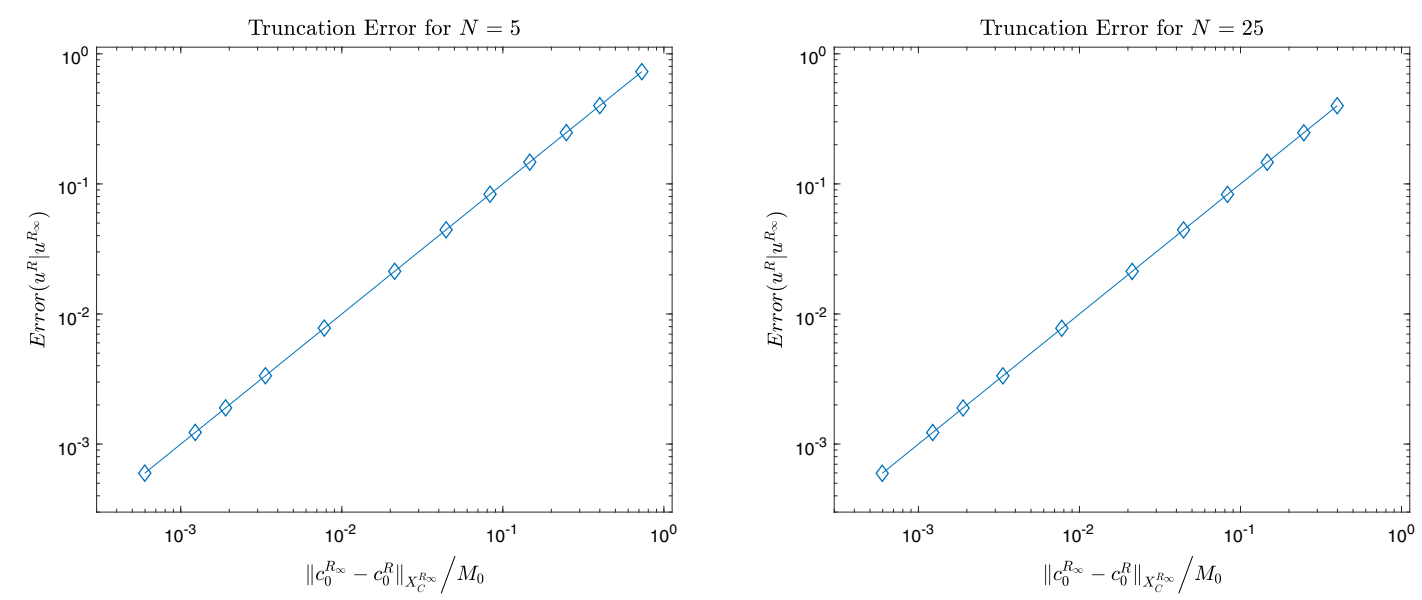

FiguRE 3 . Truncation convergence as $R \rightarrow R_{\infty}$ starting from a negative exponential initial mass distribution, for $N=5$ (left) and $N=25$ (right).

the theoretically derived value (7.6). Once again the full numerical results for all experimental configurations may be found in Appendix A of [4].

To summarise the results of this section, for the cases examined we were able to empirically confirm the analytical results established earlier in the paper. The numerically generated solutions were seen to converge as $h \searrow 0$, with the discretisation error seen to be $\mathcal{O}(h)$. We also observed the truncated solutions $\boldsymbol{u}^{R}$ converging to the full solution as we let the truncation parameter $R \rightarrow \infty$, with the rate of this convergence being governed by the initial truncation error $\left\|c_{0}-c_{0}^{R}\right\|_{X_{C}}$.

We now consider the implications of these results for the efficient use of the numerical scheme. Given a mixed fragmentation problem of the type specified by (1.1) and (1.2), the total error in our approximate numerical solutions can be bounded by the sum of the discretisation error and the truncation error. These two sources of error must be balanced in order to provide the most accurate numerical solutions for a given computational cost.

\section{Conclusions}

In this article we introduced a numerical scheme for the approximate solution of a truncated version of a mixed discrete-continuous fragmentation model. The scheme was based upon a finite volume discretisation of the modelling equation for the continuous component.

The resulting numerical approximations were first shown to be nonnegative and to conserve mass, provided the underlying mesh satisfied certain constraints. Following this we established the weak convergence of a subsequence of our approximations, as the mesh size parameter $h$ was decreased to zero. The resulting limit was then shown to provide a weak solution to the truncated model.

By relating the scalar-valued weak formulation of our truncated model to an equivalent weak formulation within a Banach space setting, we were able to apply the theory of operator semigroups to the analysis of the numerically obtained weak solution. This approach enabled us to establish a one-to-one relationship between any scalar and Banach-space-valued weak solutions. Under suitable constraints, the Banach-space-valued weak solution was shown to provide the unique strong solution to the associated abstract Cauchy problem, in the process establishing the uniqueness of the original scalar-valued weak solution. Additionally, as a further consequence of this linkage, the scalar weak solution was shown to be a differentiable classical solution, a class of regularity not ordinarily established for such numerically obtained solutions. The use of these semigroup meth- 
ods allowed for the straightforward analysis of the truncated solutions, enabling us to determine the truncation error; and establish their convergence as the truncation point $R \rightarrow \infty$.

The study was concluded by conducting a range of experiments with a test model; under varying model parameter choices and mesh refinements, we experimentally established that the error in our numerical solutions was $\mathcal{O}(h)$. Furthermore we were able to empirically demonstrate the convergence of the truncated solutions as the truncation point $R \rightarrow \infty$, whilst also verifying the theoretical value derived for the associated truncation error.

Acknowledgements. This work was supported by the UK Engineering and Physical Sciences Research Council [EP/J500495/1 03].

\section{REFERENCES}

[1] M. Aizenman and T.A. Bak, Convergence to equilibrium in a system of reacting polymers. Comm. Math. Phys. 65 (1979) $203-230$.

[2] W. Arendt, C.J.K. Batty, M. Hieber and F. Neubrander, Vector-valued laplace transforms and Cauchy Problems. In: Monographs in Mathematics. Birkhauser Verlag (2001).

[3] H. Babovsky, On a Monte Carlo approach to the Smoluchowski equations. Monte Carlo Methods App. 5 (1999) 1-18.

[4] G. Baird, Mixed discrete-continuous fragmentation equations. Ph.D. thesis, University of Oxford (2017). https://ora.ox.ac. uk/objects/uuid:311da0da-6801-4120-9129-d95786a153b6.

[5] G. Baird and E. Süli, A mixed discrete-continuous fragmentation model. J. Math. Anal. App. 473 (2019) $273-296$.

[6] J. Banasiak, On an extension of Kato-Voigt perturbation theorem for substochastic semigroups and its application. Taiwanese J. Math. 5 (2001) 169-191.

[7] J. Banasiak, Transport processes with coagulation and strong fragmentation. Discrete Contin. Dyn. Syst. B 17 (2012) $445-472$.

[8] J. Banasiak and L. Arlotti, Perturbations of Positive Semigroups with Applications. Springer-Verlag, London (2006).

[9] J. Banasiak and W. Lamb, On the application of substochastic semigroup theory to fragmentation models with mass loss. J. Math. Anal. Appl. 284 (2003) 9-30.

[10] J. Banasiak and W. Lamb, Coagulation, fragmentation and growth processes in a size structured population. Discrete Contin. Dyn. Syst. Ser. B 11 (2009) 563-585.

[11] J. Banasiak and W. Lamb, Analytic fragmentation semigroups and continuous coagulation-fragmentation equations with unbounded rates. J. Math. Anal. App. 391 (2012) 312-322.

[12] J. Banasiak and W. Lamb, The discrete fragmentation equations: semigroups, compactness and asynchronous exponential growth. Kinetic Related Models 5 (2012) 223-236.

[13] J. Banasiak and W. Lamb, On the existence of moments of solutions to fragmentation equations. J. Math. Anal. App. 413 (2014) 1017-1029.

[14] J. Banasiak, W. Lamb and M. Langer, Strong fragmentation and coagulation with power-law rates. J. Eng. Math. 82 (2013) 199-215.

[15] J. Banasiak, L.O. Joel and S. Shindin, Analysis and simulations of the discrete fragmentation equation with decay. Math. Methods Appl. Sci. 41 (2018) 6530-6545.

[16] J.C. Barrett and J.S. Jheeta, Improving the accuracy of the moments method for solving the aerosol general dynamic equation. J. Aerosol Sci. 27 (1996) 1135-1142.

[17] P.N. Blair, W. Lamb and I.W. Stewart, Coagulation and fragmentation with discrete mass loss. J. Math. Anal. Appl. 329 (2007) 1285-1302.

[18] J.P. Bourgade and F. Filbet, Convergence of a finite volume scheme for coagulation-fragmentation equations. Math. Comp. 77 (2008) 851-882.

[19] J.A. Canizo, Evolution equations in a Banach space (2006). http://canizo.org/tex/evolution.pdf.

[20] M.E. Costas, M. Moreau and L. Vicente, Some analytical and numerical solutions for colloidal aggregation with fragmentation. J. Phys. A: Math. General 28 (1995) 2981-2994.

[21] P. Degond and M. Engel, Numerical approximation of a coagulation-fragmentation model for animal group size statistics. Netw. Heterogeneous Media 12 (2017) 217-243.

[22] P. Degond, J.-G. Liu and R.L. Pego, Coagulation-fragmentation model for animal group-size statistics. J. Nonlinear Sci. 27 (2017) 379-424.

[23] J. Drazkowska, F. Windmark and C.P. Dullemond, Modeling dust growth in protoplanetary disks: the breakthrough case. Astron. Astrophys. $\mathbf{5 6 7}$ (2014) A38.

[24] A. Eibeck and W. Wagner, An efficient stochastic algorithm for studying coagulation dynamics and gelation phenomena. SIAM J. Sci. Comput. 22 (2000) 802-821.

[25] K.J. Engel and R. Nagel, One-Parameter Semigroups for Linear Evolution Equations. Springer, New York (2000).

[26] F. Filbet, An asymptotically stable scheme for diffusive coagulation-fragmentation models. Commun. Math. Sci. 6 (2008) $257-280$. 
[27] F. Filbet and P. Laurençot, Numerical simulation of the Smoluchowski coagulation equation. SIAM J. Sci. Comput. 25 (2004) $2004-2028$.

[28] A.F. Filippov, On the distribution of the sizes of particles which undergo splitting. Theory Probab. App. 6 (1961) 275-294.

[29] I. Fonseca and G. Leoni, Modern Methods in the Calculus of Variations: $L^{p}$ Spaces. Springer New York (2007).

[30] L. Forestier-Coste and S. Mancini, A finite volume preserving scheme on nonuniform meshes and for multidimensional coalescence. SIAM J. Sci. Comput. 34 (2012) B840-B860.

[31] F. Guias, A Monte Carlo approach to the Smoluchowski equations. Monte Carlo Methods App. 3 (1997) 313-326.

[32] A. Johansen, F. Brauer, C. Dullemond and H. Klahr, A coagulation-fragmentation model for the turbulent growth and destruction of preplanetesimals. Astron. Astrophys. 486 (2008) 597-611.

[33] J. Kumar, J. Saha and E. Tsotsas, Development and convergence analysis of a finite volume scheme for solving breakage equation. SIAM J. Numer. Anal. 53 (2015) 1672-1689.

[34] R. Kumar, Numerical analysis of finite volume schemes for population balance equations. Ph.D. thesis, Otto-vonGuericke University Magdeburg (2010). https://www.researchgate.net/publication/50870176_Numerical_analysis_for_ finite_volume_schemes_for_population_balance_equations.

[35] S. Kumar and D. Ramkrishna, On the solution of population balance equations by discretization-I. A fixed pivot technique. Chem. Eng. Sci. 51 (1996) 1311-1332.

[36] R. Kumar, J. Kumar and G. Warnecke, Moment preserving finite volume schemes for solving population balance equations incorporating aggregation, breakage, growth and source terms. Math. Models Methods Appl. Sci. 23 (2013) 1235-1273.

[37] R. Kumar, J. Kumar and G. Warnecke, Convergence analysis of a finite volume scheme for solving non-linear aggregationbreakage population balance equations. Kinet. Related Models 7 (2014) 713-737.

[38] W. Lamb, Existence and uniqueness results for the continuous coagulation and fragmentation equation. Math. Meth. Appl. Sci. 27 (2004) 703-721.

[39] P. Laurençot, Weak compactness techniques and coagulation equations. In: Vol. 2126 of Evolutionary Equations with Applications in Natural Sciences. Lecture Notes in Math. Springer International Publishing (2015) 199-253.

[40] E.H. Lieb and M. Loss, Analysis. Graduate Studies in Mathematics. American Mathematical Society (2001).

[41] A.C. McBride, A.L. Smith and W. Lamb, Strongly differentiable solutions of the discrete coagulation-fragmentation equation. Phys. D: Nonlinear Phenom. 239 (2010) 1436-1445.

[42] E.D. McGrady and R.M. Ziff, Shattering transition in fragmentation. Phys. Rev. Lett. 58 (1987) $892-895$.

[43] D.J. McLaughlin, W. Lamb and A.C. McBride, An existence and uniqueness theorem for a coagulation and multiplefragmentation equation. SIAM J. Math. Anal. 28 (1997) 1173-1190.

[44] D.J. McLaughlin, W. Lamb and A.C. McBride, A semigroup approach to fragmentation models. SIAM J. Math. Anal. 28 (1997) 1158-1172

[45] R. Nagel, Towards a matrix theory for unbounded operator matrices. Math. Z. 201 (1989) 57-68.

[46] M. Nicmanis and M.J. Hounslow, A finite element analysis of the steady state population balance equation for particulate systems: aggregation and growth. Comput. Chem. Eng. 20 (1996) S261-S266.

[47] A. Pazy, Semigroups of Linear Operators and Applications to Partial Differential Equations. Springer, New York (1983).

[48] S. Qamar and G. Warnecke, Solving population balance equations for two-component aggregation by a finite volume scheme. Chem. Eng. Sci. 62 (2007) 679-693.

[49] S. Qamar, G. Warnecke and M.P. Elsner, On the solution of population balances for nucleation, growth, aggregation and breakage processes. Chem. Eng. Sci. 64 (2009) 2088-2095.

[50] J. Saha, N. Das, J. Kumar and A. Bück, Numerical solutions for multidimensional fragmentation problems using finite volume methods. Kinet. Related Models 12 (2019) 79-103.

[51] A.P. Smirnov, S.A. Matveev, D.A. Dmitry, A. Zheltkov and E.E. Tyrtyshnikov. Fast and accurate finite-difference method solving multicomponent Smoluchowski coagulation equation with source and sink terms. Proc. Comput. Sci. 80 (2016) 2141-2146.

[52] L. Smith, W. Lamb, M. Langer and A.C. McBride, Discrete fragmentation with mass loss. J. Evol. Equ. 12 (2012) $181-201$.

[53] I.W. Stewart, A global existence theorem for the general coagulation-fragmentation equation with unbounded kernels. Math. Methods Appl. Sci. 11 (1989) 627-648.

[54] J. Voigt, On substochastic $C_{0}$-semigroups and their generators. Trans. Theo. Stat. Phys. 16 (1987) $453-466$.

[55] R.M. Ziff, Kinetics of polymerization. J. Stat. Phys. 23 (1980) 241-263. 